\title{
Jovian magnetospheric and auroral effects of the SL9 impacts
}

\author{
By WING-HUEN IP \\ Max-Planck-Institut für Aeronomie, D-37191 Katlenburg-Lindau, Germany
}

The collisions of comet Shoemaker-Levy 9 with Jupiter have produced many surprising auroral and magnetospheric phenomena. The energy released during the passage of the cometary dust comas through the jovian magnetosphere and at atmospheric explosion could lead to impulsive particle acceleration, enhanced radial diffusive transport, and the establishment of field-aligned current systems connecting the comet impact sites to their respective magnetic conjugate points. Some of the observed effects such as the abrupt increase of decimetric radio emission, the excitation of infrared $\mathrm{H}_{3}^{+}$emissions and mid-latitude auroral emission in the ultraviolet, could be interpreted within the framework of these mechanisms. Several auroral features like the $\mathrm{X}$-ray outbursts and short-term variations in the UV emissions are more puzzling and require further observation of jovian auroral dynamics in these wavelength ranges in coordination with the Galileo mission.

The important thing is to be there when the picture is painted.

-John Minton

\section{Introduction}

The collisions of Comet Shoemaker-Levy 9 with Jupiter in the third week of July 1994 have opened a new chapter in cometary physics and the study of Jupiter. The hypervelocity impacts of these comet nuclei at Jupiter caused many spectacular effects in the upper atmosphere and ionosphere. Very dynamic phenomena were also observed in the Jovian auroral zones and magnetosphere during the impact week. In a certain sense, the study of the magnetospheric and auroral effects caused by the atmospheric impacts of Comet Shoemaker-Levy 9 is complicated. Not only were the energetic phenomena observed closely coupled to the energy release processes in the jovian upper atmosphere and ionosphere, they could also be affected by the plasma interaction of the dust coma as well as the global dynamics of the jovian magnetosphere. Both of these topics are new in the sense that we have only an incomplete understanding of the physical effects involved. Furthermore, unlike the spectacular fireballs created by atmospheric explosions, it is difficult in some cases to identify the observed auroral features, without any ambiguity, as being the signatures associated with comet impacts. With this said, a comprehensive review of the major results will be attempted by taking a three-step approach. In the first part, we shall briefly describe the general aspects of the Jovian magnetosphere and the cometary dust coma which are important in data interpretation. This is followed by a summary of observations relevant to auroral and magnetospheric effects made at different wavelengths (e.g., radio, infrared, UV and X-rays). Theoretical ideas and models proposed to explain the many exciting and challenging observations described in preliminary reports are then described.

\subsection{The jovian magnetosphere}

In the case of the jovian magnetosphere, perhaps the most important things to know are that Jupiter has a rather fast rotation period $(P=9 \mathrm{hr} 55 \mathrm{~min} 29.7 \mathrm{~s})$ and that its 
(a) NORTH

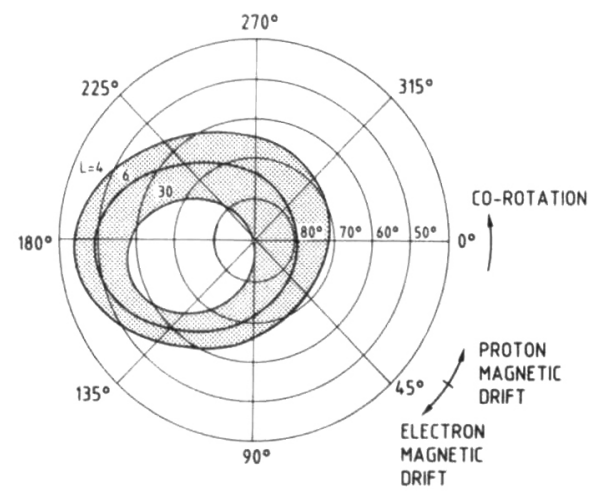

(b) SOUTH

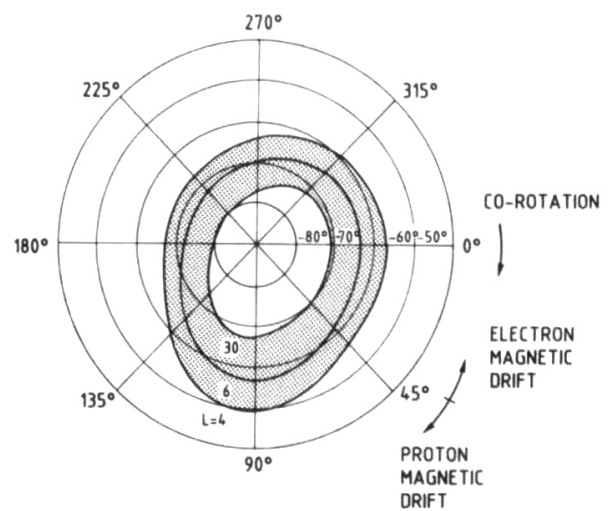

FIGURE 1. $\mathrm{L}=$ constant drift shell contours in the northern hemisphere (a) and southern hemisphere (b) of Jupiter. Adapted from Acuna et al. (1983).

magnetic field at the planetary surface is the strongest among all planetary objects. A combination of the fast rotation and a large intrinsic magnetic field results in a basic difference between the jovian magnetosphere and Earth's magnetosphere. That is, while the solar wind interaction plays a dominant role in determining the overall plasma flow pattern in the terrestrial case, the jovian magnetosphere is mainly controlled by the corotational electric field so that the plasma flow velocity in a large part of the magnetosphere is in the azimuthal direction (Brice \& Ioannidis 1970; Belcher 1983).

Another important point is that the chemical composition of the jovian magnetosphere is a mixture of the solar wind plasma and the oxygen and sulfur ions with their origin traced back to the volcanic gas of Io. A thermal plasma disc of $\mathrm{O}^{+}, \mathrm{O}^{2+}, \mathrm{S}^{+}, \mathrm{S}^{2+}$ and $\mathrm{S}^{3+}$ ions forms in the vicinity of Io. This plasma structure, called Io plasma torus (IPT), has it peak density of 3000 electrons $\mathrm{cm}^{-3}$ at $L \approx 5.6$ ( $L$ is the equatorial radial distance in units of the jovian radius, $R_{J}$ ) and is one of the most prominent features in the jovian system in optical ( $N a$ D lines and SII at $6716 \AA$ and $6731 \AA$ ) and UV radiation (Broadfoot et al. 1979; Brown et al. 1983). The radiative energy of the UV emissions from the jovian aurora and the IPT is derived from the mass loading effect of the newly ionized ions (Broadfoot et al. 1979; Dessler 1980) and probably also from other sources (Shemansky 1988).

$\mathrm{X}$-ray emissions from the polar regions were monitored by the Einstein and ROSAT space observatories long before the SL9 impacts (Metzger et al. 1983; Waite et al. 1994). The X-ray radiation shows a significant level of north-south asymmetry with strong enhancement near the north pole. Furthermore, such radiation, which is believed to be generated by the $\mathrm{K}$-shell emission from the precipitating oxygen and sulfur ions, has a significant rotational dependence with its brightness peaking near the System III Longitude $\left(\lambda_{I I}\right) 180^{\circ}-200^{\circ}$ (Waite et al. 1994, 1995). To a certain extent, this has to do with the fact that Jupiter's magnetic dipole moment is inclined to the planetary rotation axis as well as offset from the planet center (Acuna et al. 1983). As a result, the northern aurora exposes itself most to Earth viewing when the central meridian longitude is at $\lambda_{I I I} \sim 180^{\circ}$; a similar effect occurs for the southern aurora when $\lambda_{I I I} \approx 90^{\circ}$ (see Fig. 1).

A similar property of longitudinal variation is shared by the UV and infrared emissions from the jovian aurora (Herbert et al. 1987; Livengood et al. 1990; Miller et al. 1995). The imaging observations of $\mathrm{H}_{3}^{+}$auroral emission in the infrared (Connerney et al. 1993) 


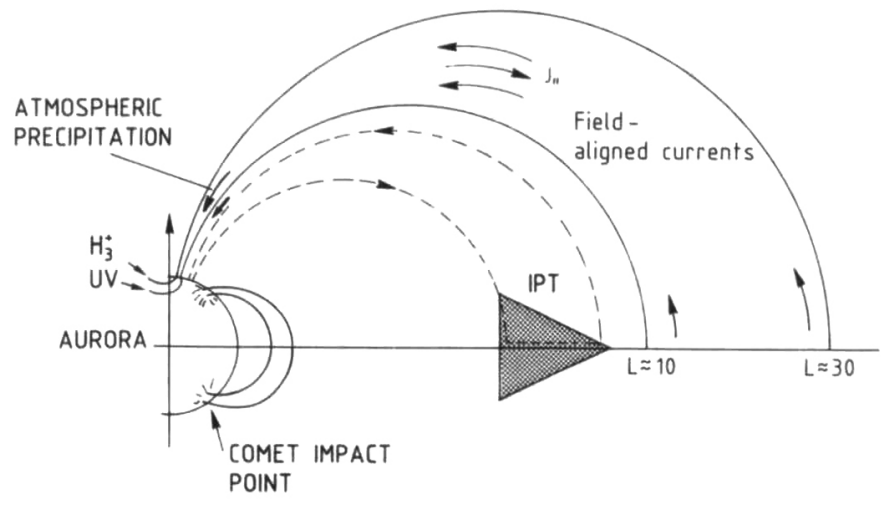

Figure 2. A sketch of the magnetic field line connection from different parts of the jovian auroral zone to the magnetosphere.

and of $\mathrm{H}$ and $\mathrm{H}_{2}$ emissions at far ultraviolet wavelengths by HST (Gerard et al. 1994a) have also shown that both of the discrete $\mathrm{H}_{3}^{+}$and UV aurora zones could be mapped to a magnetospheric region at $L>30$ (or even until reaching the magnetopause) while the more diffuse UV emissions detected by IUE and Voyager is mapped to the outer IPT region-where pitch angle scattering of the energetic ions could have the effect of producing the auroral emissions equatorward of the $\mathrm{H}_{3}^{+}$and the discrete UV aurora (Livengood 1990, Gerard 1994a). During its high latitude crossing of the jovian system, the Ulysses spacecraft observed a system of field-aligned currents connecting the polar auroral zones to the outer magnetosphere at $L \approx 20-30$ with a total current of $10-90 \times 10^{6} \AA$ (Dougherty et al. 1993). The measurements of field-aligned beams of energetic electrons and ions by the particle instruments on Ulysses (Lanzerotti et al. 1993) are also consistent with the scenario that the jovian auroras are excited by atmospheric precipitation of the currentcarrying particles. Prangé et al. (1995a) reviewed the collisional excitation effects of the $\mathrm{H}_{2}$ Werner bands in the FUV and concluded that the energy of the primary population of precipitating particles is likely to be $\approx 40-150 \mathrm{keV}$ for electrons and $2-10 \mathrm{MeV} /$ nucleon for ions. Figure 2 illustrates the present understanding of the regions where auroral emissions have their sources.

Also of special interest to the present discussion is that long term monitoring of the jovian UV auroral activity by IUE has established that the average auroral brightness profile in general does not vary by more than a factor of 2-3. Because of the lack of spatial resolution, the IUE observations could not provide information on whether any of the brightness variations are associated with morphological changes in localized areas. A recent coordinated observation program of simultaneous measurements using IUE and the Faint Object Camera on HST recorded a sudden brightness change in the northern aurora by a factor $>10$ over a time period of $<20$ hours (Gerard et al. 1994b). The maximum value of the total $\mathrm{H}_{2}$ emission $(\approx 6 \mathrm{MR})$ and the short time scale suggested that disruption of the global current system of the jovian magnetosphere because of an interplanetary disturbance might have been the underlying cause of this event. According to Gerard et al. (1994b), an interesting consequence of the large energy deposition rate $\left(\approx 1 \mathrm{~W} / \mathrm{m}^{2}\right)$ from particle precipitation is that strong thermospheric winds could be generated because of the intense atmospheric heating. This effect will be a familar theme in our discussions of the SL9 atmospheric impacts. 


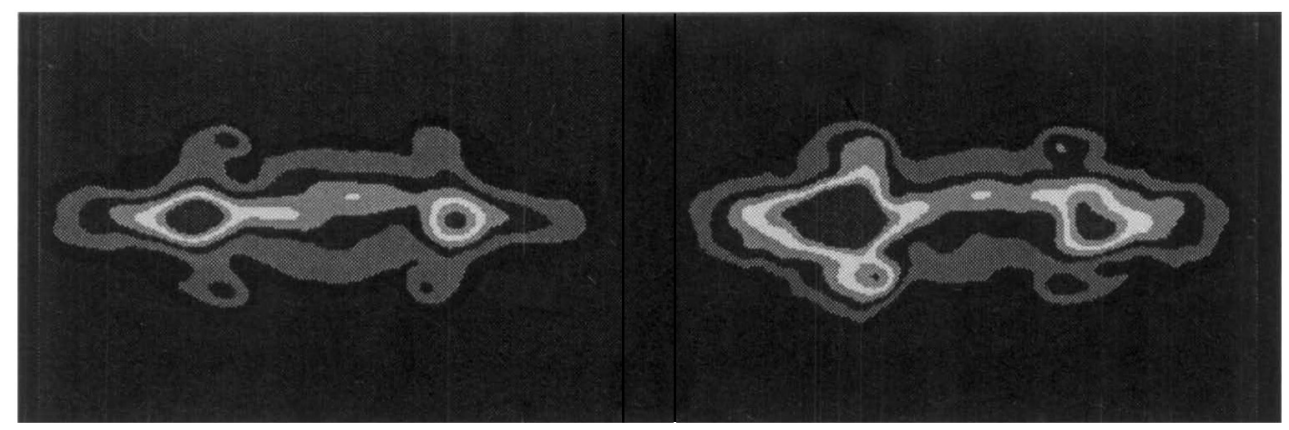

FIgURE 3. VLA $20 \mathrm{~cm}$ radio images of Jupiter at $\lambda_{I I I} \approx 118^{\circ}$ at two 1994 UT dates: (a) June 24:03 h UT (before the comet impacts), and (b) July 20:03 UT (during the impact week). The two images are on the same intensity scale. The peak value in image (a) is $\approx 1700 \mathrm{~K}$ and $\approx 2500 \mathrm{~K}$ in image (b). The full beam width at half power is $\approx 0.3 R_{J}$. From de Pater et al. (1995).

Jupiter has long been known to be a strong source of decimetric and decametric radio emissions (see Goldstein and Goertz 1983; de Pater and Klein 1989). As a result, the information concerning their short-term and long-term variations is also most complete and telling as far as the responses to the SL9 impacts are concerned. The decimetric emissions from the synchrotron radiation of relativistic electrons in the inner trapped radiation belt are particularly well documented. As depicted in Fig. 3, the 2D intensity map shows a maximum at $L \approx 1.5$ with the brightness concentrated near the equatorial region. This pancake-like structure originates from the flattening of the pitch angle distribution of the $\mathrm{MeV}$ electrons as a consequence of their inward diffusion while conserving the first adiabatic invariant. The displacement and tilting of the planetary magnetic moment leads to a rocking of the synchrotron radiation structure as Jupiter rotates. Detailed comparisons of such pre-impact data and measurements obtained during and after the impact week will prove to be very valuable in analyses of magnetospheric effects.

\subsection{The dust coma}

The best source of information on the properties of the dust coma of SL9 have come from the HST Wide Field Planetary Camera observations by Weaver et al. (1995). In the inner coma the brightness profiles display a $\rho^{-1.16}$ dependence out to $1^{\prime \prime}$ (where $\rho$ is the radial distance from the comet nucleus) which means that there might have been continuous dust emission from the nucleus surface. These observers estimated that the dust production rate should be on the order of $5 \mathrm{~kg} \mathrm{~s}^{-1}$ for some of the brighter nuclei. Only upper limits of the corresponding gas production rates were given. For example, according to $\mathrm{H}$. Rickman (priv. comm., 1995) the production rate of $\mathrm{CO}$-which was possibly the dominant sublimating gas - was found to be below that of $\mathrm{P} /$ SchwassmannWachmann 1 at $5.8 \mathrm{AU}$ the value of which is $\mathrm{Q}(\mathrm{CO}) \sim 5 \times 10^{28}$ molecules $^{-1}$ (Senay and Jewitt 1994; Crovisier et al. 1995), and the upper limit on the $\mathrm{H}_{2} \mathrm{O}$ production rate was found to be $\sim 10^{27}$ molecules $\mathrm{s}^{-1}$ by Weaver et al. (1995). Thus, it is by no means certain that the dust coma must be accompanied by any significant level of gas sublimation. As an alternative, an electrostatic blowoff mechanism could play a role in ejecting small dust particles from the bare nucleus (Mendis et al. 1981; Ip 1984). 


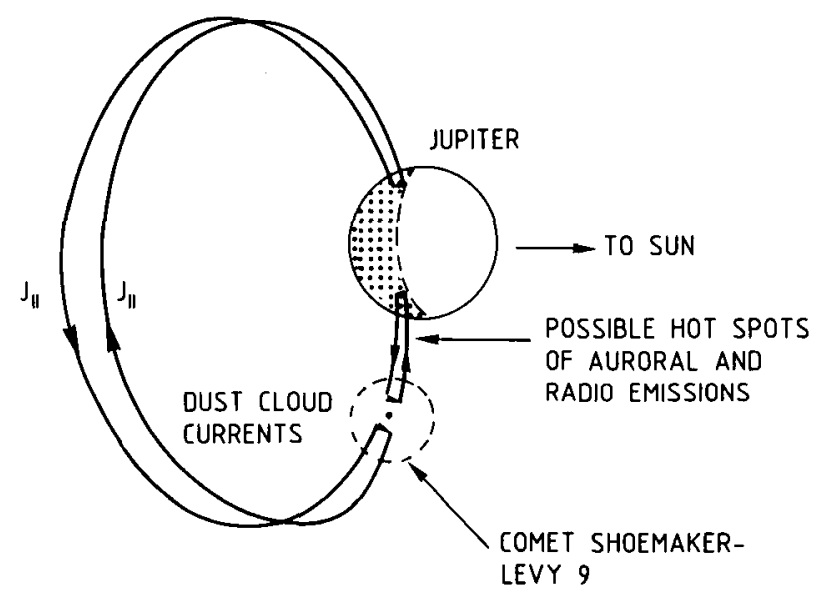

FIGURE 4. A global structure of the field-aligned current system that might have been established between the coma (or the atmospheric impact site) of comet SL9 and the jovian ionosphere. From Ip and Prangé (1994).

Because the cometary dust has a very fragile structure, it is possible that electrostatic force on charged grains could lead to fragmentation when subject to the surface charging effect of energetic electrons (Hill \& Mendis 1980). It was on this basis that Dessler \& Hill (1994) suggested that as the SL9 comet comas entered the jovian magnetosphere, the electrostatic fragmentation process could make the dust coma visible because of the enhanced cross sectional area of the dust fragments. However, no significant changes to the coma structures were observed except for a short burst of $\mathrm{Mg}^{+}$ emission followed by a three-fold increase in continuum emission when the $\mathrm{G}$ fragment was $54 R_{J}$ away from Jupiter (Weaver et al. 1995). The brightness increase in continuum emission lasted about 8 minutes and then returned to the quiescent level 20 minutes after the outburst. This time variation might in part be due to the dispersal of the cloud of fragmented dust of submicron size by the Lorentz force. For particles of micron size in the extended coma the Lorentz force would still be effective in causing some deviations of their orbital motion from that of the comet nucleus as it moves toward Jupiter (Horanyi 1994). A careful study of such a dynamical effect is still to be carried out.

The dust coma could interact with the jovian magnetosphere in several ways depending on the effective conductivity of the charged dust cloud and other factors. If the dust coma is partially conducting because of the development of an ionosphere, the magnetospheric interaction will be cometary-like and a current system will be generated between the comet and the jovian ionosphere possibly triggering decametric emissions (Kellogg 1994; Ip and Prangé 1994). On the other hand, if the relative motion between the charged dust particles and the magnetospheric plasma is unimpeded inside the dust coma, the corresponding differential motion between the negatively charged dust and the corotating ions would also lead to a field-aligned current system as shown in Fig. 4. It is in this manner that magnetospheric disturbances in the southern hemisphere associated with a comet impact could be transmitted to the magnetic conjugate point in the northern hemisphere along the magnetic field line.

To summarize, some of the auroral and magnetospheric effects are expected to be arranged in terms of field-aligned current systems connecting the impact site to its magnetic 


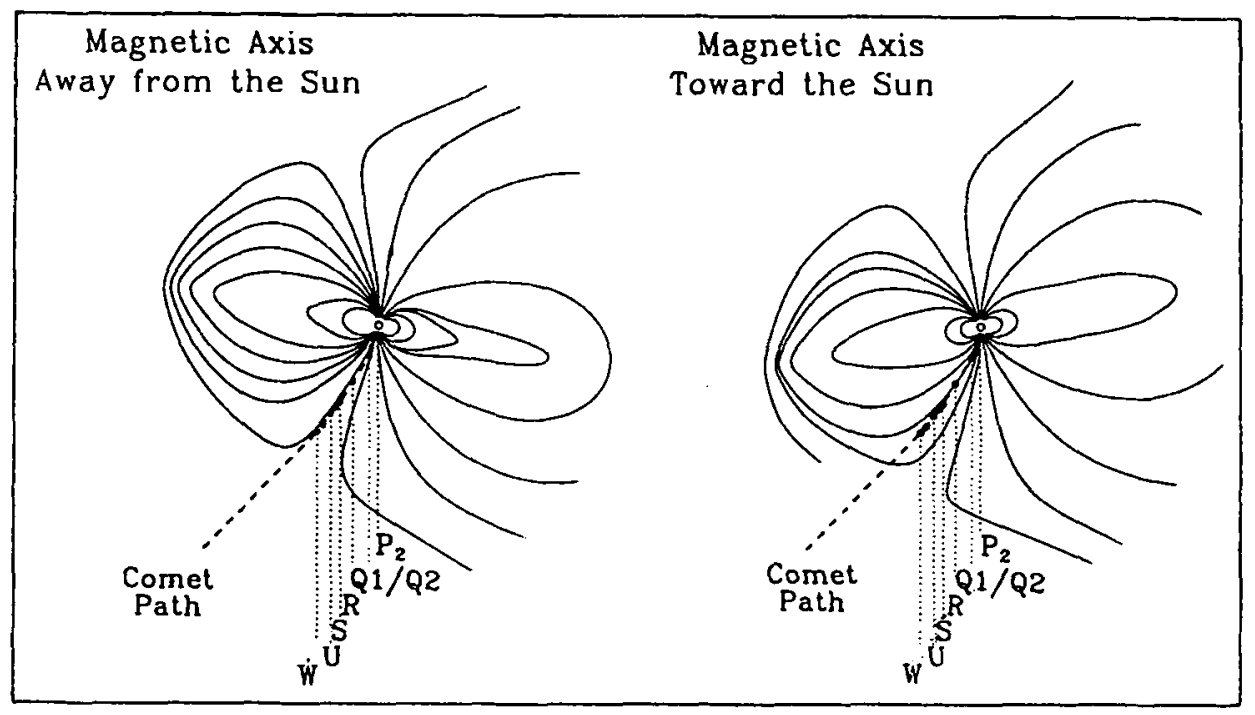

FIGURE 5. A sketch of the orbital spiral traced by comet SL9 in the rotating jovian magnetosphere. Before its final atmospheric impact, the comet and its dust coma could sweep across a wide range of $L$ shells covering regions with closed or open magnetic field lines. From Prangé et al. (1995b).

conjugate point. However, such magnetic comnection is complicated by the inclination and rotation of the jovian magnetosphere. For example, the position of the comet fragments could alternate between the region of closed magnetic field lines and the region with the planetary magnetic field lines opened to the interplanetary magnetic lines (Prangé et al. 1995a). These two basic configurations are depicted in Fig. 5. As we shall see some observed features could indeed be described by the simple picture outlined here. However, there are many unexpected surprises-as is typical of magnetospheric plasmas.

\section{Observations}

The world-wide coverage of the magnetospheric effects of the SL9 impacts has been quite phenomenal and extremely fruitful. These comprehensive data sets dealing with a variety of temporal variations will be very useful in the diagnosis of SL9-related effects and the physics of the inner radiation belt of Jupiter. But it is really the fortuitous combination of the coordinated microwave measurements by several major radio telescopes, observations of the $\mathrm{H}_{3}^{+}$emission at $3.5 \mu \mathrm{m}$ by infrared telescopes (i.e., UKIRT at Mauna Kea and the 3.5-m NTT at La Silla), ultraviolet observations using the International Ultraviolet Explorer (IUE) satellite, the Extreme Ultraviolet Explorer (EUVE), the Hubble Space Telescope, and finally the X-ray observations by ROSAT which provides much of the synergism. If the SL9 impacts were to have taken place five years earlier, our information on the magnetospheric and auroral effects would have been limited to microwave and IUE observations and perhaps nothing else. With this in mind, we will summarize the highlights of these very unique results. 


\subsection{Microwave observations}

As discussed in the Introduction, the synchrotron radiation of relativistic electrons in the energy range of $10-20 \mathrm{MeV}$ peaks at about $1.5 R_{J}$; this means the narrow jovian ring located between 1.72 and $1.81 R_{J}$ would cause absorption collision and energy degradation of these relativistic electrons as they diffuse inward from larger radial distance (de Pater and Goertz 1990). Besides the particulate matter in the jovian ring, magnetospheric electrons could be depleted by dust particles injected from the comet comas into the jovian magnetosphere. Thus if the collisional interaction of magnetospheric electrons with the comet dust is significant, a reduction of microwave radiation would probably be observable (de Pater 1994; Ip 1994). Even though closer scrutiny of the mass budget showed that the increase in the dust population, if any, would be quite small and little decrease in the synchrotron radiation would be expected (Dessler and Hill 1994), the general expectation before the comet impacts was still that some reduction in the microwave emissions might occur. The fact that sharp increases in the brightness fluxes were seen at different wavelengths throughout the impact week therefore came as a major surprise (Dulk et al. 1995; Leblanc and Dulk 1995; de Pater et al. 1995; Klein et al. 1995). According to Klein et al. (1995) such changes were unprecedented in the 23-year history of the NASA-JPL Jupiter patrol. There is, therefore, little doubt that they are impactrelated. A summary of the temporal variations of the brightness fluxes between 6-cm and $90-\mathrm{cm}$ wavelengths is given in Fig. 6 . The radio emissions all tend to reach peak values at the end of the impact week. The increases were wavelength dependent, with the amplitude varying from about $10 \%$ at $70-90 \mathrm{~cm}$ to about $45 \%$ at $6 \mathrm{~cm}$.

It is important to note that the decay time scales $\left(t_{d}\right)$ for the brightness flux increases were also found to be different for different wavelengths. For the first few months, the 6 -cm component had $t_{d} \approx 250$ days, the $13-\mathrm{cm}$ had $t_{d} \approx 125$ days and $t_{d} \approx 76$ days for the 21-cm component (Klein et al. 1995). As a result, most of the microwave emissions have not returned to pre-impact values several months later except for the 70-90 cm component, which appeared to drop below its preimpact value forty days after the impact week; curiously enough, there also appeared to be a drop in the brightness flux immediately after the impact week as observed by the Westerbork Synthesis Radio Telescope (WSRT).

Leblanc \& Dulk (1995) reported that there existed a significant longitudinal asymmetry in the brightness increase at least until July 28 with most of the enhancement confined to one hemisphere with $\lambda \approx 100^{\circ}-240^{\circ}$, e.g., the active sector of the jovian magnetosphere (Vasyliunas and Dessler 1981). On the other hand, de Pater et al. (1995) concluded that the impact-induced asymmetry lasted no more than four days on the basis of the VLA observations. The dispersion time scale of $10 \mathrm{MeV}$ electrons as a result of azimuthal drift is on the order of two days. A confinement of the brightness enhancement to the magnetic active sector for a time interval much longer than this drift period would require pitch angle scattering of the trapped electrons and/or a continuous injection of new electrons into this region (I. de Pater, priv. comm. 1995). A detailed analysis of the time variations of the longitudinal asymmetry of the microwave emission enhancement will be essential to the understanding of the source region and storage of the relativistic electrons generated by the cometary impacts.

A comparison of the brightness fluxes at different times shows that the spectral shape of the electron energy distribution might have been hardened or alternatively, the pitchangle distribution of the $\mathrm{MeV}$ electrons was isotropized following the comet impacts (Bolton and Thorne 1995; de Pater et al. 1995). Before the impacts, the radio spectrum follows a simple power law of $\mathrm{S} \sim \nu^{-\alpha}$, with $\alpha \approx 0.10$ at $\nu>20 \mathrm{~cm}$ and $\alpha \approx 0.4$ at 

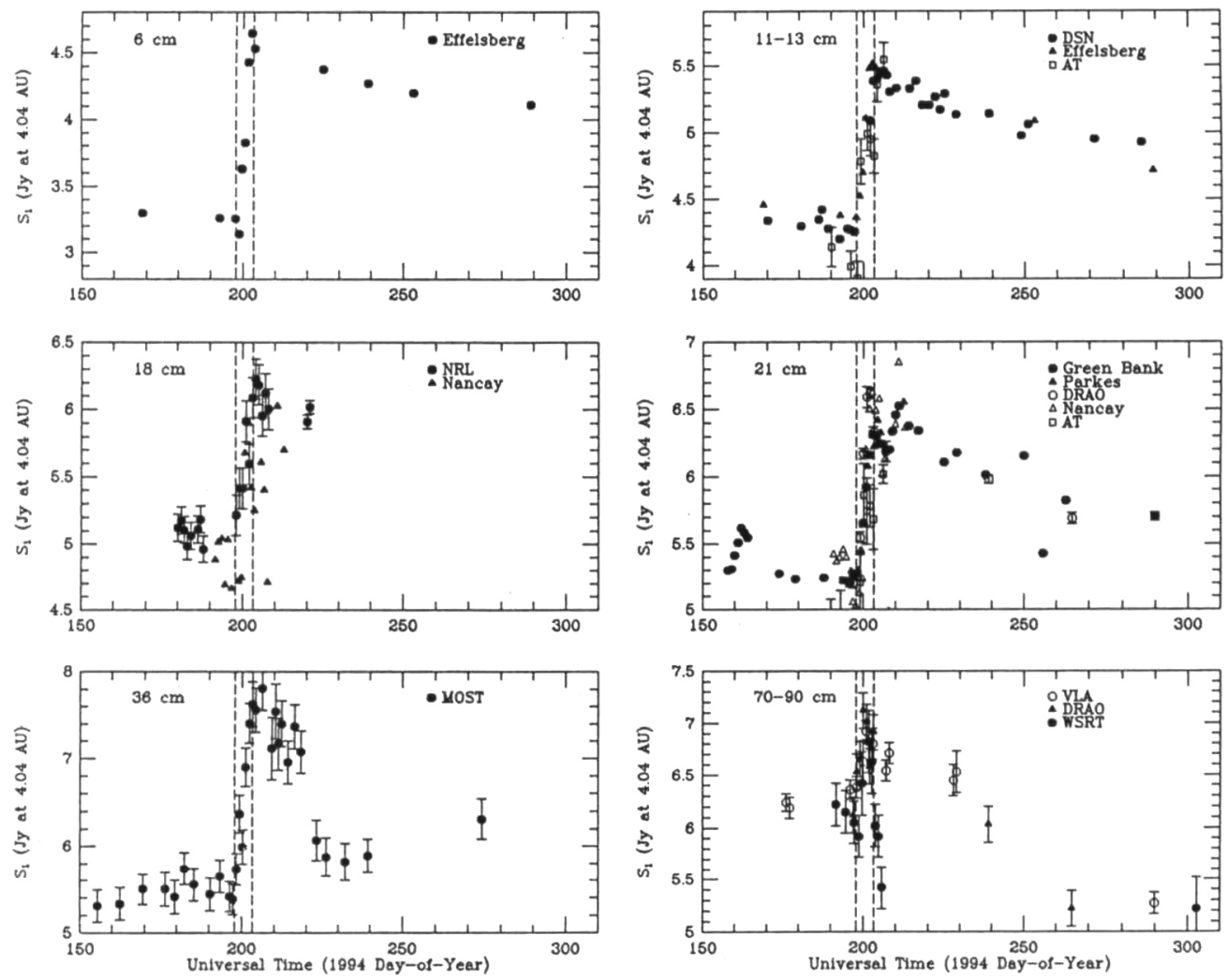

FIgURE 6. Jupiter's nonthermal flux densities between $6 \mathrm{~cm}$ and $70-90 \mathrm{~cm}$. From de Pater et al. (1995).

$\lambda<20 \mathrm{~cm}$. After the impact week, there was a conspicuous maximum at $36 \mathrm{~cm}$ and $\lambda \approx 0.30$ for $\lambda>20 \mathrm{~cm}$.

The observed effect of radio spectrum hardening could be caused by several mechanisms including (a) impact-driven radial diffusion of the radiation belt electrons initially located at larger radial distance, (b) pitch-angle scattering of the pre-existing electron population to larger pitch angles at which mirroring points the corresponding synchrotron radiation would be intensified, and (c) in-situ acceleration of electrons to energies $>10 \mathrm{MeV}$ (see Fig. 7). In the first case, electron energization because of conservation of the first adiabatic invariant (i.e., $E^{2} / B=$ const.) during the impulsive inward diffusion could be a very effective mechanism in producing an increase in the synchrotron radiation (Ip, $1995 \mathrm{a}$ ). Because the radiation intensity $S \approx E^{2} B^{2}$ and hence $S \approx 1 / L^{9}$ in a dipole field geometry; an inward shift of the relativistic electrons by just $\Delta L \approx 0.05$ at $L \approx 1.5$ would lead to an increase of the brightness flux by as much as $30 \%$. This effect is consistent with the decrease of the radial separation between the intensity peaks from $2.92 R_{J}$ to $2.82 R_{J}$ observed by Leblanc and Dulk (1995) at $13 \mathrm{~cm}$. The $20 \mathrm{~cm}$ VLA images also showed an inward movement of the intensity peak near $\lambda_{I I I} \sim 60^{\circ}-110^{\circ}$ by $0.2 R_{J}$ (de Pater et. al. 1995). 


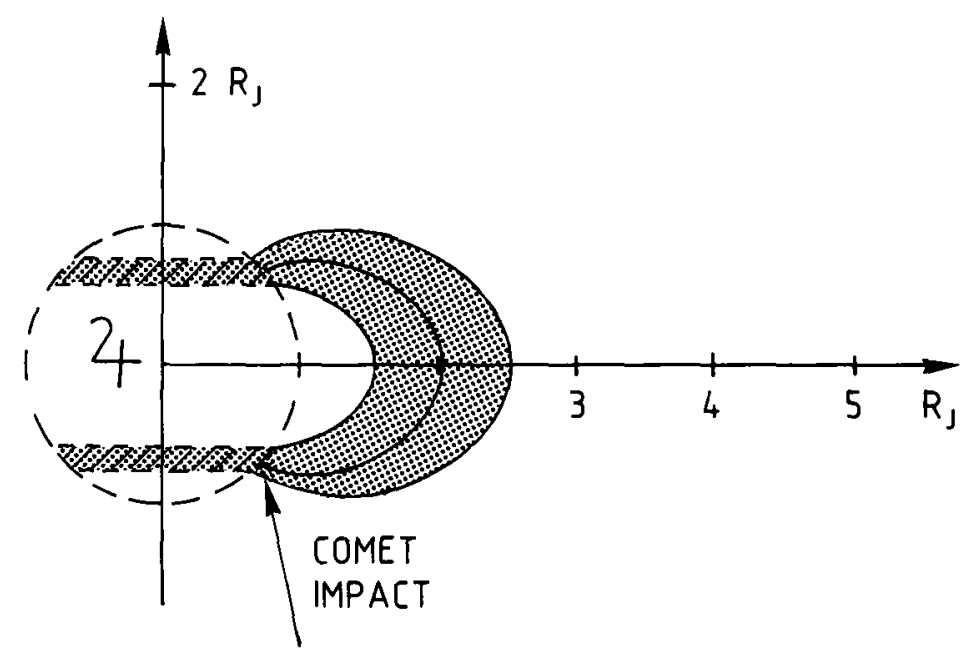

FIgURE 7. A schematic view of the magnetic flux tube with enhanced neutral wind dynamo effect and possibly pitch angle scattering process caused by the comet impact-driven plasma wave turbulence.

Conservation of the first adiabatic invariant would require that the pitch angle distribution become more and more flattened as the radiation belt particles diffuse inward to regions of stronger magnetic field. Thus, we would expect a higher concentration of the synchrotron radiation near the magnetic equator if the impact-driven radial diffusion mechanism is the cause of the increase in the microwave radiations. However, Klein et al. (1995) reported that the observed change in the magnetic latitude beaming curves during the impact week suggests an increase in the emission at higher magnetic latitudes. For this to happen, significant level of pitch angle scattering of the locally trapped electrons [e.g., mechanism (b)] would have to take place (Bolton and Thorne 1995). Because the determination of beaming curves could be affected by the longitudinal asymmetry of the microwave brightness distribution detected by de Pater et al. (1995) and Leblanc \& Dulk (1995), a detailed examination of the 2D images of the radio maps would be required to separate these two effects.

Finally, in-situ electron acceleration in the magnetic flux tubes connected to the locations of atmospheric explosions in Jupiter remain a possibility (Brecht et al. 1995). This particular mechanism would probably instigate a sequence of step function-like increases in the microwave emissions following individual comet impacts. Examination of high time resolution data of radio measurements would be very useful in resolving this particular issue (see Section 3.3).

\subsection{Infrared observations}

Several programs of infrared observations during the comet impact week were planned. Of major importance in the study of jovian auroral activities are (a) the imaging and spectroscopic measurements at $3.53 \mu \mathrm{m}$ of the $\mathrm{H}_{3}^{+}$emission by the University College London group (Miller et al. 1995) using the CGS4 on the United Kingdom Infrared Telescope and the NSFcam imager on NASA's Infrared Telescope Facility at Mauna Kea, (b) the near-IR spectrometric measurements using the 3.5-m ESO New Technology Tele- 
scope at La Silla (Schulz et al. 1995), and (c) the CASPIR on the ANU $2.3 \mathrm{~m}$ telescope at the Siding Spring Observatory, Australia (McGregor et al. 1995).

The ESO measurements in the $3.5 \mu \mathrm{m}$ region $(3.501 \mu \mathrm{m}-3.566 \mu \mathrm{m}, \lambda / \Delta \lambda \sim 1700)$ between July 22 and 31, 1994, showed intense $\mathrm{H}_{3}^{+}$emissions over the positions of the comet impact sites in the southern hemisphere ( $44^{\circ} \mathrm{S}$ latitude). Mapping of the jovian disk was performed by Schulz et al. (1995) by displacing the slit from south to north in several steps. Anomalous $\mathrm{H}_{3}^{+}$emissions at $44^{\circ} \mathrm{N}$ latitude were found with their longitudes in good correlation with their counterparts in the southern hemisphere (Fig. 8). This effect showed that after a comet collision, a population of ionizing particles was injected at the impact site and travelled to its magnetic conjugate point on the opposite hemisphere. What is interesting is that the mid-latitude $\mathrm{H}_{3}^{+}$emission in the northern aurora was considerably intensified at the later phase of the observations (July 31) until the flux values of $\mathrm{H}_{3}^{+}$in the northern auroral region were around ten times higher than in the southern aurora; at the same time, the $\mathrm{H}_{3}^{+}$emissions near the impact sites had faded to a relatively low value. Discrete bright spots of IR emission in the northern hemisphere were also observed in the 3-4 $\mu \mathrm{m}$ CASPIR images taken at the Siding Spring Observatory after the $\mathrm{K}$ and $\mathrm{G}$ impacts (McGregor et al. 1995). The IR spot observed in the image made 45 min after the $\mathrm{K}$ collision appears to be closely related to the intense UV emission arcs detected by HST at nearly the same time interval (Clarke et al. 1995); and both emissions triggered by the comet impact displayed rather short time scale $(\approx$ one hour) in the brightness decrease. The transient nature of the mid-latitude auroral activity detected by CASPIR is therefore not consistent with the long-term effect reported by Schulz et al. (1995). This discrepancy remains to be resolved by detailed analysis of the impact-related IR obesrvations and continuous monitoring of the $\mathrm{H}_{3}^{+}$emission in the jovian disk.

Before comet impacts, the north/south (N/S) ratio of the $\mathrm{H}_{3}^{+}$auroral emissions ratio was $\approx 1-3$, which is within the norm of the average auroral variabilities. Sudden unusual variations of the auroral emission were reported by all three groups. For example, the UKIRT and IRTF observations by Miller et al. (1995) showed that between 25 and 27 July the N/S ratio was seen to increase to $\approx 6-10$ before returning to the average value of about 3 . This change was caused in part by the enhancement of the northern emission and in part by a suppression of the southern one. Another important result from Miller et al. (1995) concerns the fact that very strong longitudinal variations were also found. The northern emission reached the highest intensity at $\lambda_{I I I}$ between $90^{\circ}$ and $270^{\circ}$ while the southern emission generally leveled off to values below the pre-impact intensities. To explain this remarkable north-south asymmetry, Miller et al. (1995) suggested that it could be due to the reduction of the ionospheric Pedersen conductivity in the southern auroral zone because of the injection of a large amount of $\mathrm{H}_{2} \mathrm{O}$ and $\mathrm{CO}$ molecules and dust of cometary origin (Cravens 1994). This would effectively shortcircuit the field-aligned current flow between the equatorial plasma sheet and the southern hemisphere but would double the electric current going into the northern part and hence the temporal variation of the $\mathrm{N} / \mathrm{S}$ ratio of the $\mathrm{H}_{3}^{+}$auroral emissions. This scenario can be further tested by searching for the putative $\mathrm{H}_{3} \mathrm{O}^{+}$ions (at $2.9 \mu \mathrm{m}$ ) in the near-IR spectra and detailed aeronomical model calculations taking into consideration the vertical distribution of dust and $\mathrm{H}_{2} \mathrm{O}$ and $\mathrm{CO}$ molecules in the jovian upper atmosphere. It is however important to note that, as will be discussed later, energetic events characterized by ultraviolet and X-ray emissions associated with the P2 and Q1/Q2 impacts have been detected in the northern auroral zone. Such effect could potentially play an important role in the generation of the $\mathrm{H}_{3}^{+}$emission in the northern aurora. 


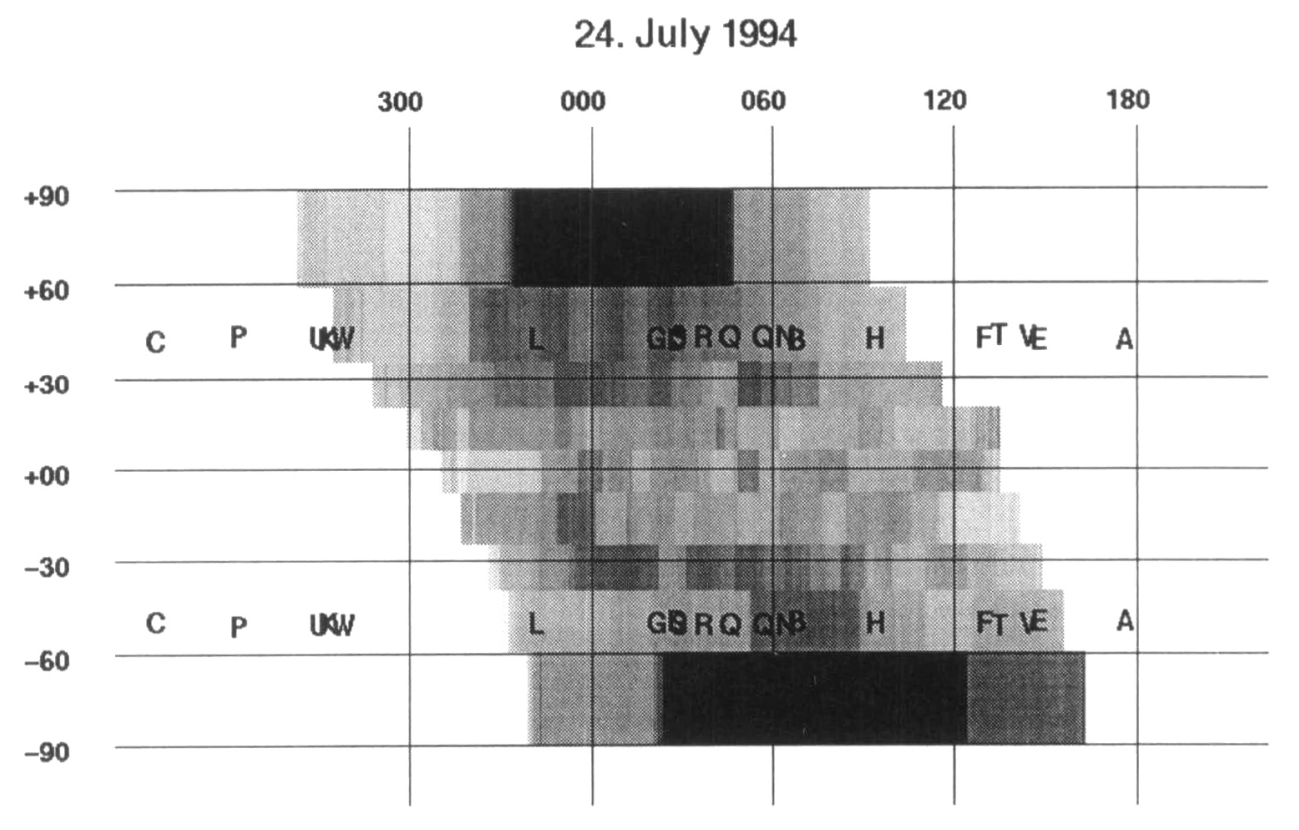

Figure 8. A map showing the spatial distribution of the $\mathrm{H}_{3}^{+}$line $(3.528-3.536 \mu \mathrm{m})$ on the jovian disk on July 24, 1994. The map was constructed by shifting the slit in 9 steps from the south pole to the north pole. The System III longitudes of the impact sites are specified in the figure. This observation was made using the IRSPEC spectrometer at the $3.5-\mathrm{m}$ New Technology Telescope at ESO, La Silla. From Schulz et al. (1995).

\subsection{Ultraviolet observations}

The IUE was employed to monitor the auroral activities of Jupiter during the impact week (Ballester et al. 1995). Three observational sessions were scheduled for the $\mathrm{K}, \mathrm{S}$ and P2 fragments each with an integration time $\sim 20$ minutes centered around the impact time. (Strong emissions were detected only for the $\mathrm{K}$ and $\mathrm{S}$ impacts). The position of the aperture was centered at the impact sites in the southern hemisphere. The exact dimension of the source region of $\mathrm{H}$ Lyman alpha and $\mathrm{H}_{2}$ emission features is limited by the full-width-half-maximum (FWHM $\approx 5^{\prime \prime}$ ) of the SWP camera. For the $\mathrm{K}$ impact, which has the brightest UV emissions, the $\mathrm{H}_{2}$ feature at $1610 \AA$ appeared to be point-like along dispersion. A diameter of $2^{\prime \prime}(7330 \mathrm{~km})$ for the emission area and an effective exposure time of 10 minutes were used to estimate the lower limit of the surface brightnesses. The derived value of a few tens of $\mathrm{kR}$ for the hydrogen emissions is in fact as large as the normal jovian auroral brightnesses (Livengood et al. 1990). The lower limit of the radiative energy output was estimated to be about $10^{21} \mathrm{ergs}$. Because of the long integration time $(\approx 10$ minutes) these UV emissions which must be of a transient nature might have a much greater brightness reaching MR levels at the initial phase of the mid-latitude auroral activities.

From fits to observed spectra (see Figure 9) with electron excitation models, it was tentatively concluded that the $\mathrm{H}_{2}$ emissions might have been caused by collisional excitation by electrons with energies $\gtrsim 20 \mathrm{eV}$ (Ballester et, al. 1995). (It is however probable 


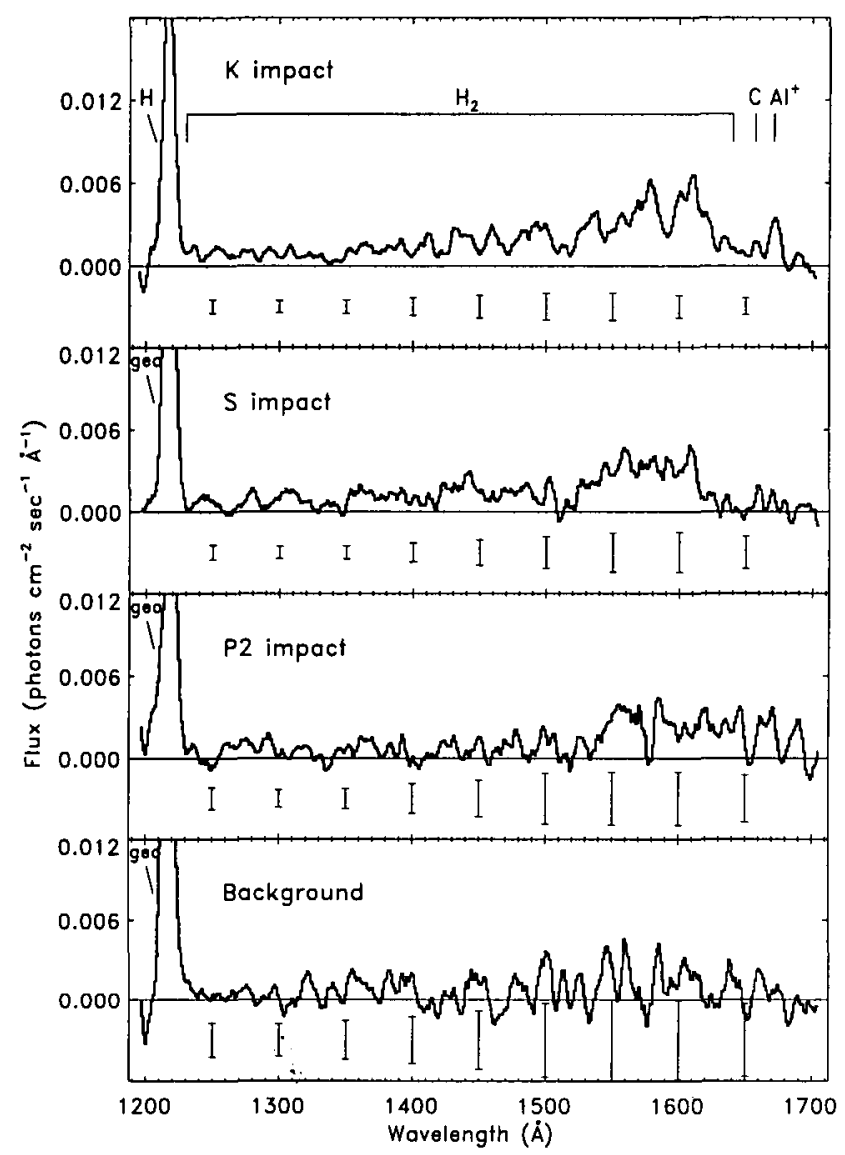

FIGURE 9. Representative IUE spectra for the K, S and P2 impacts and background observations, respectively from top to bottom. The absolute spectral fluxes are lower limits evaluated with the full exposure times. From Ballester et al. (1995).

that the primary precipitating particles could have much higher energies.] In addition to the $\mathrm{H}$ Lyman alpha and the $\mathrm{H}_{2}$ Lyman- and Werner-band emissions, spectral features of $\mathrm{C} 1657 \mathrm{~A}$ and possible $\mathrm{Al}^{+} 1671 \mathrm{~A}$ could be tentatively identified. Both $\mathrm{C}$ and $\mathrm{Al}$ atoms could have originated from the cometary material deposited in the jovian atmosphere.

It was unfortunate that the HST observation of the $\mathrm{K}$ impact was not scheduled at the same time as the IUE measurements. Otherwise, the immediate response of the jovian atmosphere at the impact site as well as at the magnetic conjugate point in the northern hemisphere would have been registered. Two HST observations were made 47 and 57 minutes after the $\mathrm{K}$ impact (Clarke et al. 1995). Figure 10 shows one of the FUV inages of discrete mid-latitude auroral emissions of about $280 \mathrm{kR}$ near the approaching limb. The two northern emission arcs covering (a) $51^{\circ} \mathrm{N}$ (latitude), $257^{\circ}-277^{\circ}$ (longitude) and (b) $56^{\circ} \mathrm{N}, 238^{\circ}-258^{\circ}$ are the most conspicuous features. Some faint emission could also be identified just south of the $\mathrm{K}$ impact site with the latitude $54^{\circ} \mathrm{S}, 275^{\circ}$ and $52^{\circ} \mathrm{S}$, $280^{\circ}$. The north and south UV emissions appeared to be connected by magnetic field geometry. However, it is important to note that no emission was detected at the northern conjugate point (at $38^{\circ} \mathrm{N}$ latitude and $269^{\circ}$ longitude) of the $\mathrm{K}$ impact site (at $43.8^{\circ} \mathrm{S}$ latitude and $279^{\circ}$ longitude). 


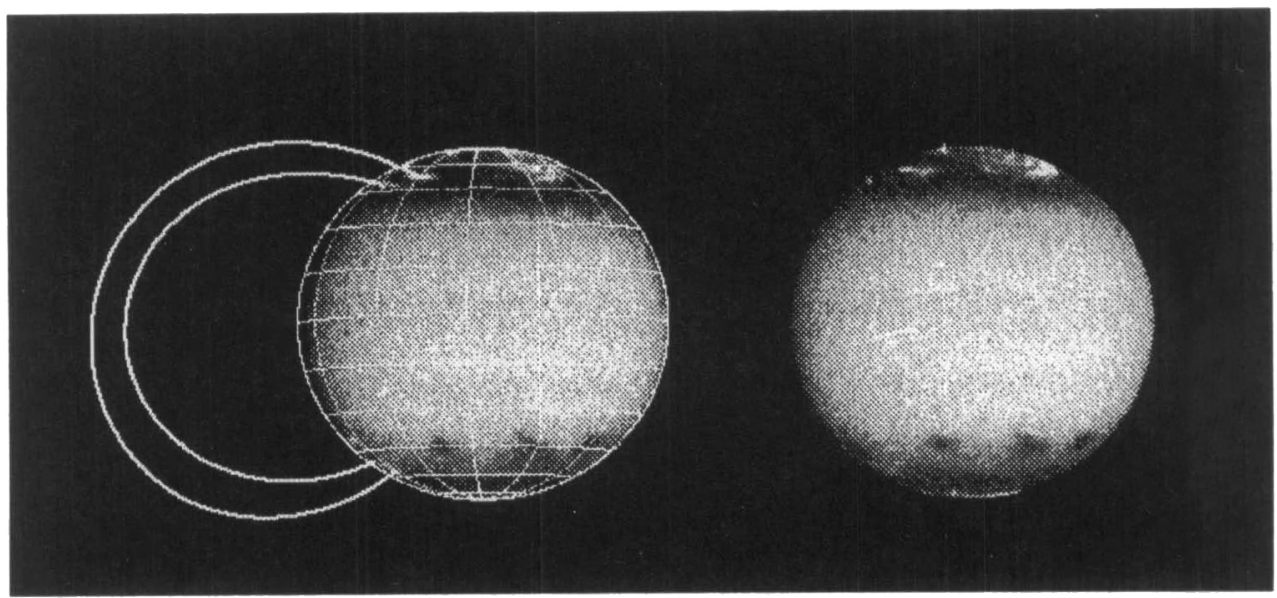

FIgURE 10. A WFPC2 FUV images from HST taken 47 and 57 min after the $\mathrm{K}$ impact (assumed at 10:24 UT), showing auroral emissions at lower latitudes than normally observed and apparently associated with the $\mathrm{K}$ impact event. Magnetic field lines from the $\mathrm{O}_{6}$ model are overplotted to show the connection of the northern emission centers to near the $K$ impact region in the southern hemisphere. The integration time of the image is 400 sec. From Clarke et al. (1995).

The cause of the observed spatial displacement is uncertain. There are three possibilities. First, the neutral wind disturbances which could be instrumental in driving a field-aligned current (FAC) system could propagate towards the poles bringing with them the FAC footprints (Hill and Dessler 1995). Second, if a population of energetic ions are accelerated in the magnetic flux tube connected to the $\mathrm{K}$ impact site, their subsequent interaction with the jovian magnetosphere could lead to a radial transport and diffusion of the particles. Finally, the particle acceleration effect need not be confined to the magnetic flux tube connected to the $\mathrm{K}$ impact site; that is, plasma disturbances could be generated along the path of the comet fragment which swept through a range of magnetic latitudes just before atmospheric collision. In this way, excitation of UV emissions at somewhat higher latitudes would be expected. There are indeed some indications of the latter process. According to Clarke et al. (1995) and Prangé et al. (1995b), two spots of significant UV emission (brightnesses $\approx 200-240 \mathrm{kR}$ ) were detected near the southern polar cap at the time of the P2 impact. These UV spots were detected twice at 20 minutes interval (hence the term "blinking aurora") at 20.590 (i.e., July 20. 14:10) and 20.603 before the actual $\mathrm{P}$ impact at 20.615 (Figure 11). At the times of the blinking auroral emissions, the $\mathrm{P}$ fragment was still about one jovian radius away from the planetary surface. By using a set of jovian magnetic field models, Prangé et al. (1995b) showed that these UV spots could possibly be magnetically connected to the Q1 and Q2 fragments which were about $7 R_{J}$ away from Jupiter. Consequently, these authors suggested that interaction of the dust coma of the Q1/Q2 fragment with the jovian magnetosphere could be responsible for the excitation of these UV emissions.

Even though the HST observations of the blinking aurora could possibly be interpreted in terms of dust coma interaction, caution must be excercised. This is because we are 


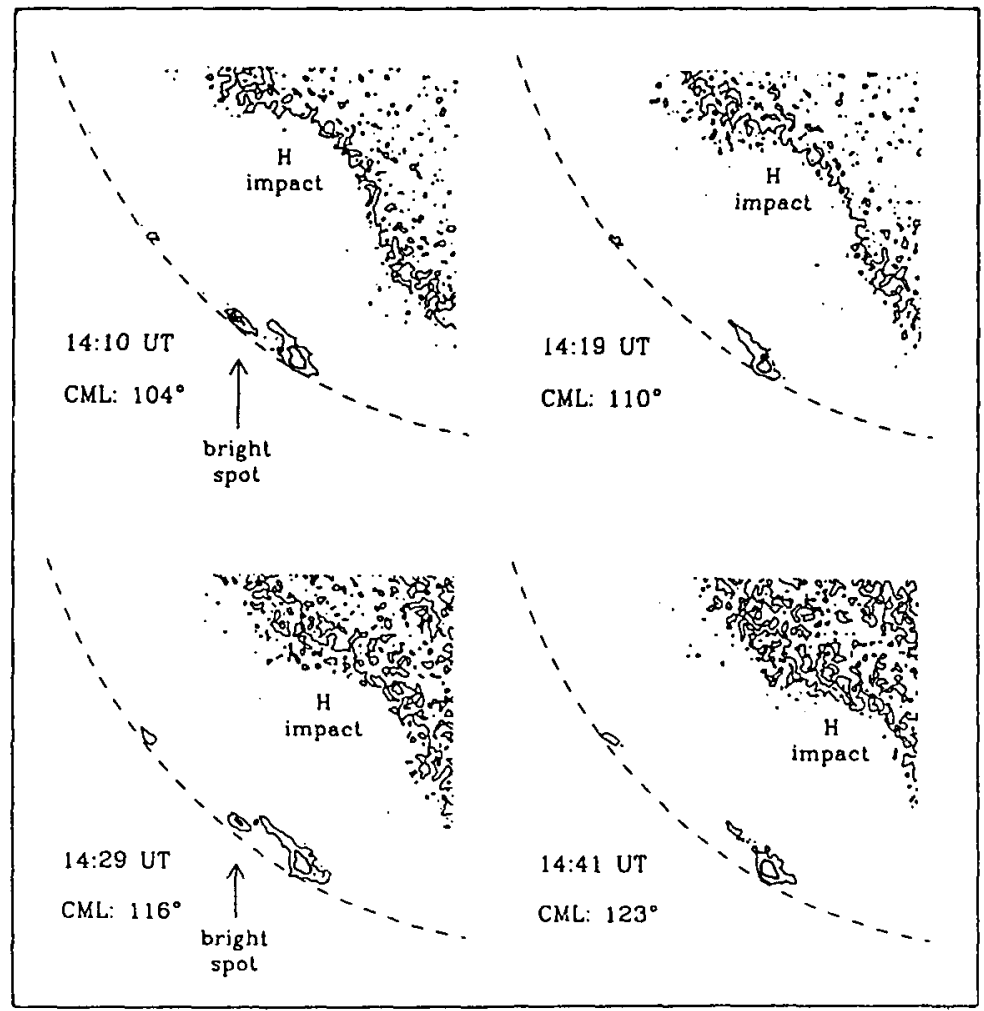

Figure 11. Four consecutive views of Jupiter's south pole from the FUV images taken with the HST WFPC2 on July 20, 1994. Isocontours are about 100 and $170 \mathrm{kR}$. The dashed line has been adjusted on the auroral oval limb gradient and is not the planet disk limb. Note the unusual bright spot on images (a) at 14:10 and (c) at 14:29 amid the emission features associated with the permanent auroral oval. From Prangé et al. (1995b).

still at a very early stage in the study of the morphology of the jovian aurora. In the few observational opportunities by HST, rather dynamic changes of the auroral features were found with the sporadic formation of auroral arcs and patchy structures in the polar cap region presumably in response to the solar wind condition (Gerard et al. 1994b; Clarke et al. 1995; J. E. P. Connerney, priv. comm., 1995). There thus exists the possibility that the presence of the "blinking aurora" might just be part of the normal variation of the jovian auroral activity and not necessary related to the comet impacts. The discovery of these interesting time-variable features during the SL9 impacts hence underlines the need for more HST observations so that the statistical behaviour of the jovian polar aurora can be better understood.

\subsection{X-ray observations}

The maximum surface brightness of the UV emissions following the $\mathrm{K}$ impact was $250 \mathrm{kR}$ in the first HST observation at $47 \mathrm{~min}$ and $180 \mathrm{kR}$ at $57 \mathrm{~min}$ after the atmospheric collision (Clarke et al. 1995). This implies a time scale of about $10 \mathrm{~min}$ for an exponential decay of the UV emission. The initial UV emission immediately after the comet im- 


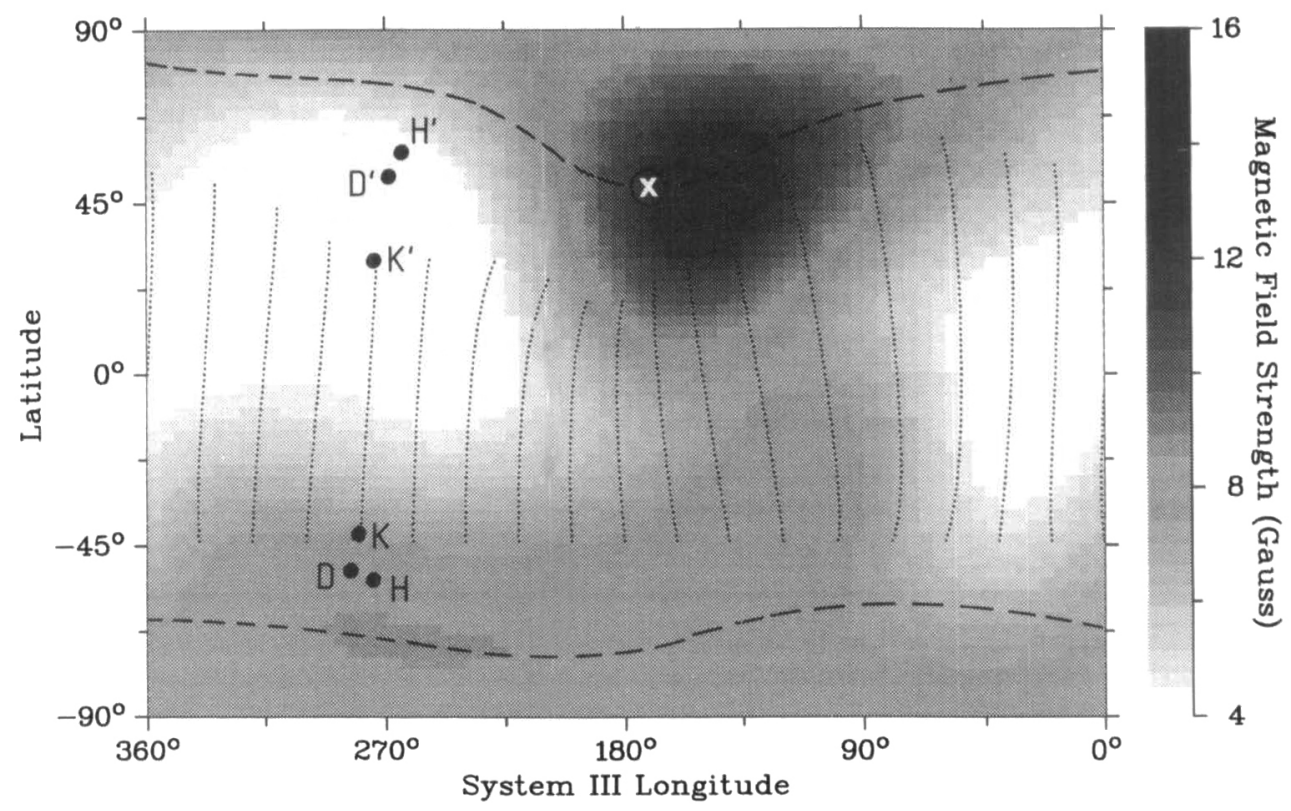

FIGURE 12. Locations of the $\mathrm{K}$ impact and its conjugate point $\left(\mathrm{K}^{\prime}\right)$ in the northern hemisphere, the low-latitude UV emissions detected by HST $\left(\mathrm{D} / \mathrm{H}\right.$ and $\left.\mathrm{D}^{\prime} / \mathrm{H}^{\prime}\right)$, and the X-ray outburst $(\mathrm{X})$ in the $\mathrm{O} 4$ magnetic model. The dotted curves (.....) represent magnetic field lines connecting from the comet impact points at latitude $=45^{\circ} \mathrm{S}$ and different System III longitudes to the corresponding conjugate points. The footprints of the Io flux tube are indicated by the dashed curves $(---)$.

pact could therefore be as high as $1200 \mathrm{kR}$, a value compatible with the lower limit determined by the IUE observations of Ballester et al. (1995). This also means that the total radiative energy output could be on the order of $10^{22}$ ergs and the corresponding mechanical energy input ten times larger. The peak power is therefore on the order of $10^{12}-10^{13} \mathrm{~W}$. However, an even more energetic event was observed by ROSAT. Using the high-resolution imager (HRI) of ROSAT, Waite et al. (1995) monitored the X-ray emission of Jupiter during the impact week. X-ray outbursts were detected in the northern hemisphere in time intervals associated with the $\mathrm{K}$ and $\mathrm{P} 2$ impacts (Figure 11). These events will be discussed in more detail in the following.

As described earlier, previous observations by the Einstein Observatory and ROSAT favoured the statistical argument that the normal X-ray emissions from the jovian aurora could be the result of $\mathrm{K}$ shell emission from precipitating energetic $(\geq 400 \mathrm{keV} / \mathrm{amu})$ sulfur and oxygen ions (Metzger et al. 1983; Waite et al. 1994). From a comparison of the BATSE measurements on the Compton Gamma Ray Observatory with the ROSAT results in the same time frame, Waite et al. (1995) reached the tentative conclusion that the heavy ions (instead of the 1-10 MeV electrons) were also responsible for the excitation of the X-ray emission at the $\mathrm{K}$ impact. In these circumstances, an input power of $2 \times 10^{13} \mathrm{~W}$ and $4 \times 10^{22}$ ergs of particle energy would be required to produce the $\mathrm{K}$ emission which lasted about $30 \mathrm{sec}$. [A similar energy budget holds for the P2 emission.] Where did these energetic ions come from? How could they be generated in such a short time and then disappear just as quickly? To address these issues we must first identify the locations of these X-ray emissions. 
The nominal pointing accuracy of HRI is 5-6 arcseconds. However, the identification and location of visible stars within the field of view of ROSAT during the SL9 observations permitted an improvement of the pointing accuracy to $3^{\prime \prime}$. This led to the positioning of the $\mathrm{K}$ impact burst to $50^{\circ} \mathrm{N}$ latitude and $\lambda_{I I I}=170^{\circ}$. While this emission peak was close to the footpoint of the Io flux tube (latitude $=50^{\circ} \mathrm{N}$ and $\lambda_{I I I}=186^{\circ}$ ), it was well separated from the magnetic conjugate point of the $\mathrm{K}$ impact site. As for timing, the impact $\mathrm{K}$ event was registered three minutes before the predicted atmospheric collision of the $\mathrm{K}$ fragment. However, the crossing of the magnetic field lines near Io's orbit by the $\mathrm{K}$ fragment took place at about $14 \mathrm{~min}$ before the atmospheric explosion. Such lack of temporal and spatial correlations has no obvious explanation if this X-ray emission was produced by the comet impact.

A similar difficulty is found with the X-ray outburst said to be associated with the P2 impact. First, the brightness enhancement detected between 15:07-15:40 UT (July 20) was seen at $\lambda_{I I I}=180^{\circ}$ and $70^{\circ} \mathrm{N}$ latitude which is completely disconnected magnetically from the P2 impact site in the southern hemisphere. Second, the X-ray emission occurred between the P2 and the Q1/Q2 impacts. There is therefore uncertainty as to which fragment (in any) should be responsible for the X-ray emission. As a matter of fact, Prangé et al. (1995b) suggested that this energetic event was caused by the magnetospheric interaction of the dust coma of the Q1/Q2 fragment as they approached Jupiter. The magnetic field mapping showed that the footprint of the Q1/Q2 fragment should be located in the proximity of $\lambda_{I I I} \sim 150^{\circ}-160^{\circ}$ at this time interval, the putative interconnection with the $\mathrm{x}$-ray burst an intriguing possibility.

The fact that both the $\mathrm{K}$ and P2-related X-ray bursts appeared at $\lambda_{I I I} \sim 180^{\circ}$ is noteworthy. Since the ROSAT X-ray data obtained in previous years indicate that the $\mathrm{X}$-ray aurora displays a pattern of longitudinal asymmetry similar to that of the UV and IR aurorae with a peak brightness near $\lambda_{I I I}=180^{\circ}-200^{\circ}$ (Waite et al. 1994), the locations of the two X-ray events suggest that they might not be directly related to the SL9 impacts, but rather only reflecting the "regular" pattern of the X-ray auroral emission. The seeming confusion in the timing and location of the outbursts has reinforced the idea that the ordinary X-ray aurora might appear in short-lived bursts with the occurrence frequency peaking near $\lambda_{I I I} \sim 180^{\circ}-200^{\circ}$. A definite answer would require long-term monitoring of the jovian X-ray aurora with high time and spatial resolutions and good pointing.

\subsection{The Io plasma torus}

Before the comet impacts several predictions were made that the jovian ionosphere would be significantly modified so that the corotational motion of the Io plasma torus (IPT) would display certain anomalous features such as a slowing down of the plasma flow (Cravens 1994). Furthermore, the possible injection of cometary dust grains into the jovian magnetosphere could lead to the addition of metallic ions to the IPT as well as absorption of the magnetospheric charged particles (Horanyi 1994; de Pater 1994; Herbert 1994). Several observational programs in the optical and UV wavelength ranges were thus planned to detect these predicted effects. In essence, no exceptional changes which could be related to the comet impacts were found. For example, the EUVE observations of the IPT luminosity reported by McGrath et al. (1995) have been shown to be consistent with no temporal changes during the impact week (Hall et al. 1995). High spectral-resolution ground-based observations by Brown et al. (1995) in a threeweek period covering the SL9 impacts have shown that the day-to-day variations in the ion densities, ion temperature, and plasma rotation velocities were not atypical of the time changes in the data obtained by identical method and instrument in previous years. 
We are therefore left with the impression that the IPT was not disturbed by the comet impacts to any significant level. Of course, the question remains as to why the energetic events leading to the X-ray bursts in the magnetic flux tube threading through the IPT and/or the enhanced radial diffusion did not leave any observable signatures.

\section{Theories}

Most of the magnetospheric and auroral effects produced by the SL9 impacts were not foreseen. This should not come as a surprise because of our lack of precise knowledge of the jovian magnetosphere and plasma-dust interaction which might have played an important role. In this regard, the observations of the comet impact effects have in fact provided us with a unique opportunity to test some of the basic theories which could be applied to the impact effects and vice versa. With this in mind, we shall consider several related theoretical topics, namely, the radial diffusion, current systems and particle acceleration mechanisms in their simplified forms.

\subsection{Radial diffusion}

The interplanetary electric field plays a dominant role in defining the convection pattern of the terrestrial magnetosphere. As a result, the radial diffusion of charged particles in the trapped radiation belt is caused by the fluctuations of the electrostatic field which, in turn, is controlled by the solar wind interaction (Schulz and Lanzerotti 1974). In the case of Jupiter, because of its large intrinsic magnetic field and rapid rotation the magnetospheric plasma flow pattern is largely determined by the corotational electric field. For this reason, the electrostatic field variations driven by the neutral wind turbulence in the upper atmosphere become important in defining the random walk motion of the magnetospheric particles. For relativistic electrons with slow gradient drift, the diffusion motion is related to the electrostatic potential fluctuations $\Delta \phi$ with time scale comparable to the orbital period of the energetic electrons in the dipole field by the following expression (Brice and McDonough 1973):

$$
D_{L L}=6.7 \times 10^{-8} L^{3}\left(\frac{\Delta \phi_{o}}{m_{e} c^{2}}\right)^{2} R_{J}^{2} / \mathrm{hr}
$$

where $\Delta \phi=\Delta \phi_{o} \cos \theta$ and $\theta$ is the latitude, $m_{e} c^{2}$ is the electron rest mass. It is interesting to note that data analyses of the Pioneer $10 / 11$ and Voyager $1 / 2$ measurements of the energetic charged particles suggested an empirical expression of $D_{L L}=D_{o} L^{3}$ with $D_{o}=4 \times 10^{-9} R_{J}^{2} \mathrm{~s}^{-1}$ (Thomsen 1979; Hood 1993; de Pater and Goertz 1994). Such an experimental value of $D_{o}$ is compatible with $\Delta \phi / m_{e} c^{2} \approx 16$ in the above equation.

For an electron to drift from dawn to dusk through a longitudinal angle of $180^{\circ}$ in a drift shell of radial distance $L$, the effective electric field would be $<E_{n}>\approx \Delta \phi_{o} / \pi L R_{J}$. Because the impact points of comet Shoemaker-Levy 9 correspond approximately to $L \approx 2$ in a centered dipole field (see later discussion), the required change in the neutral wind speed $V_{n}\left(=E_{n} / B\right)$ is on the order of $110 \mathrm{~m} \mathrm{~s}^{-1}$ per $5 \mathrm{hr}$. This means that if a transient neutral component could be generated as a result of atmospheric explosions following the comet impacts and that the wind magnitude at the magnetic flux tube of $L \approx 2$ could reach a value of about $100-200 \mathrm{~m} \mathrm{~s}^{-1}$, the radial diffusion coefficient in the vicinity of the $L$ shell encompassing the impact sites could accordingly be increased by a factor of two (see Fig. 7). By assuming a step-function-like change in the value of $D_{o}$ :

$$
\begin{aligned}
& D_{o}=9.3 \times 10^{-9} \text { for } 1.5<L<2.5 \\
& 4.6 \times 10^{-9} \quad \text { elsewhere }
\end{aligned}
$$




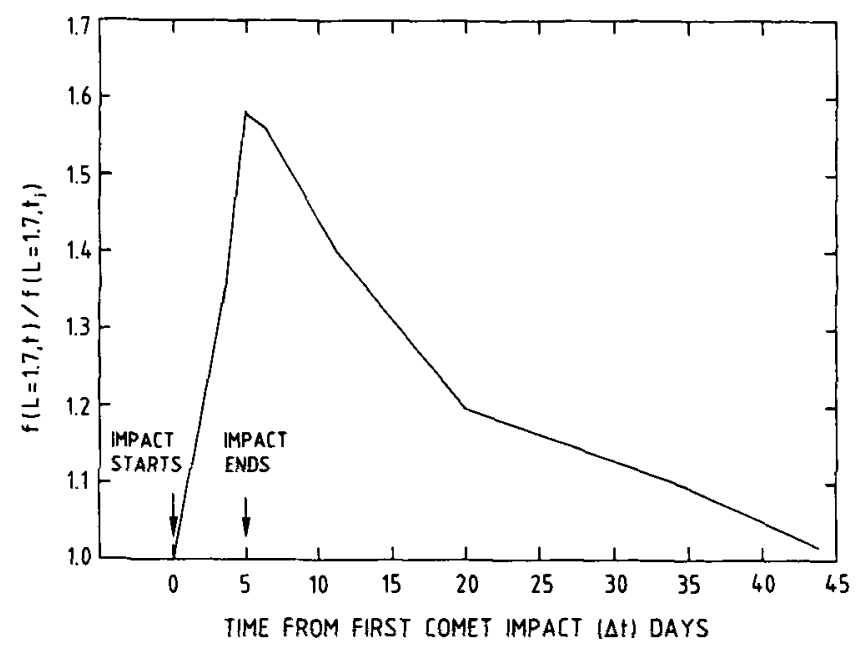

FIGURE 13. The time history of the normalized phase space density of energetic electrons (and the corresponding flux-density of synchrotron radiation) at $L \sim 1.7$ according to the impact-driven radial diffusion model.

and the further assumption that immediately after the last comet impact on July 22 , the radial diffusion coefficient would return to the normal value given by $D_{0}=4.6 \times 10^{-9}$, it can be shown that the phase space density of the $\mathrm{MeV}$ electrons at $L \approx 1.7$ would increase by $60 \%$ (Ip 1995a). An example of the time history of the flux-density of the synchrotron radiation is illustrated in Fig. 13. The time scale for the decay of the flux density enhancement could be estimated to be about 40 days. In this simple model treating the one-dimensional diffusion problem, the betatron acceleration effect as a result of the conservation of the first adiabatic invariant was not taken into account. In a more realistic approach, both radial diffusion and the energy variations should be considered simultaneously (see de Pater and Goertz 1994). The energy-dependence and hence frequency-dependence of the time variations of the microwave emissions could then be compared with the observations described in Sec. 2 .

Another important fact, of course, is that the displacement of the dipole moment from the planetary center could have caused additional temporal and longitudinal variations. As depicted in Fig. 14, magnetic field lines connected to comet impact sites with different $\lambda_{I I I}$ values could map to very different radial distances at the magnetic equator. For example, the magnetic flux tube of the A impact reaches a maximum distance of $1.5 R_{J}$ while that of the $Q$ impact has a value of about $2 R_{J}$. This means relativistic electrons injected inward after the $A$ impact would probably cause immediate enhancement of the synchrotron radiation near the brightness center of the microwave emission. On the other hand, there would be a diffusion time scale of about 10 days for the electrons injected at $L \approx 2$ to reach $L=1.5$ (by which time the azimuthal drift effect would have dispersed the electrons longitudinally around Jupiter). This might be one possible explanation why a strong concentration of microwave emissions was observed during the impact week (Dulk et al. 1995; de Pater et al. 1995).

\subsection{Current systems}

The momentum and energy in a magnetospheric system can be transferred from one region to another via electric current flows. If a current system could be established 


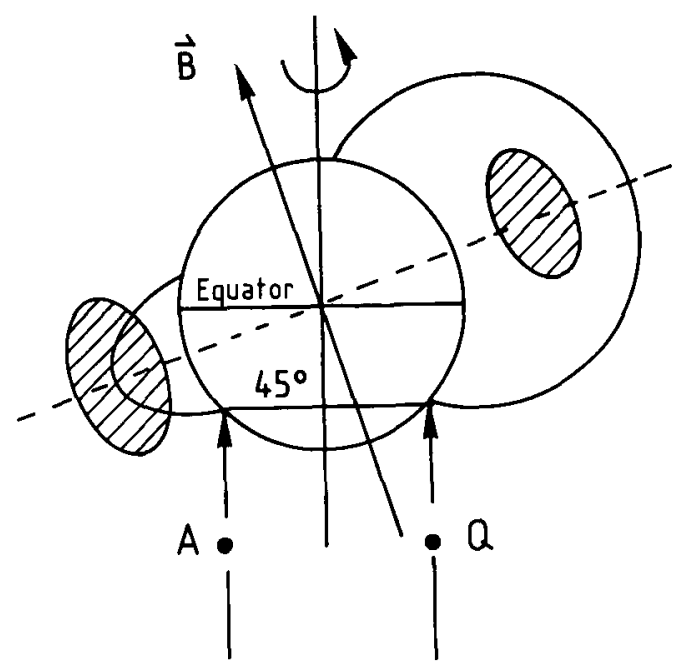

FIgURE 14. A schematic diagram showing the synchrotron radiation belts and the L-shells in the meridian plane of the magnetic field. The latitude line of $45^{\circ} \mathrm{S}$ is at the footprint of $L \approx 1.5$ at $\lambda_{I I I} \sim 200^{\circ}$ (for impact A), and at the $L \approx 2.5$ and $\lambda_{I I I} \sim 20^{\circ}$ (for impact Q). From Dulk and Leblanc (1994).

between the comet coma and the jovian ionosphere, it is conceivable that the kinetic energy from the interaction of the comet with the magnetosphere could be dissipated in the topside ionosphere in the form of auroral or radio wave emission. Following this line of argument, Farrell et al. (1994) and Ip and Prangé (1994) have independently suggested that the relative motion between the charged dust particles in the cometary coma and the corotating plasma could generate a field-aligned current system mapping from the comet to the two footpoints of the magnetic flux tube (see Fig. 4). Prangé et al. (1995b,c) have further suggested that such a process could be a source mechanism for the occurrence of the "blinking aurora" in the northern hemisphere observed by HST. It should be noted that, in addition to the possibility of field-aligned currents, MHD waves and plasma waves might be generated by the passage of the charged dust cloud. This aspect of dust-plasma interaction could indeed be of potential importance.

At atmospheric explosion, a substantial amount of the impact energy of the nucleus fragment was released in the form of shock wave and large-scale wind motion. The frictional interaction between the neutral wind and the ionized gas in the vicinity of the impact site (which might also be instrumental in causing an enhanced level of radial diffusion) could then act as an ionospheric dynamo. Following this line of thinking, Hill \& Dessler (1995) have suggested that the atmospheric expansion associated with the impact plume of the $\mathrm{K}$ fragment could maintain a field-aligned current system connecting the dynamo region to the conjugate foot point of the corresponding magnetic flux tube. Kinetic energy released in the comet impact could therefore be transferred to the opposite hemisphere which acted as a load in the circuit. If the parallel current density $\left(j_{\|}\right)$ exceeds a certain value, voltage drops or electrostatic double layers would form along the magnetic field lines. Electrons and ions could thus be accelerated to high energies causing ultraviolet and $\mathrm{X}$-ray emissions (if the voltage drop is large enough) at atmospheric precipitation-just as in the case of the terrestrial aurora. In this way, the particle 


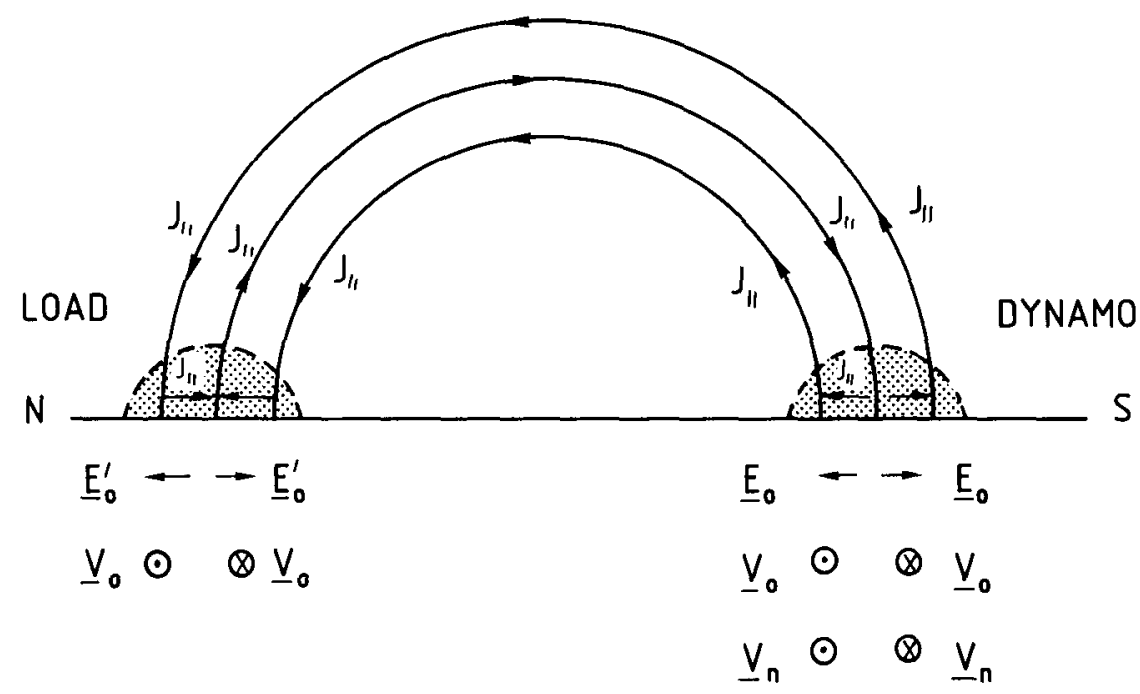

Figure 15. An idealized model of the magnetic field coupling between the dynamo region to the energy dissipation region in the opposite hemisphere. The ionospheric dynamo mechanism is driven by a neutral wind shear of velocity $\left(V_{n}\right)$. The maximum energy transfer is reached when the plasma derift velocity $\left(V_{o}\right)$ is half the value of $V_{n}$.

acceleration effect could play an important role in the dissipation of the energy stored in the current system. The IUE observed intense UV emissions at the $\mathrm{K}$ and $\mathrm{S}$ impacts with radiative energies in the $\mathrm{H}_{2}$ Lyman- and Werner-band and Lyman-alpha exceeding $10^{21}$ ergs (Ballester et al. 1995). Also the mid-latitude UV emissions observed between 47 and 57 min after the K impact by HST (Clarke et al. 1995; Prangé et al. 1995a) have a total radiative power output on the order of $3 \times 10^{11} \mathrm{~W}$ (J. T. Clarke, priv. comm., 1995). The questions are then, what might be the required magnitude of the current flow and whether this current could maintain a voltage drop large enough to excite the observed UV emissions.

The actual situation is rather complicated because of the three dimensional geometry and time evolution of the neutral wind system (see Hill and Dessler 1995). To make some order of magnitude estimates, we shall consider the highly simplified case of a steady state atmospheric dynamo mechanism in which shear flows with wind speeds $\left(V_{n}\right)$ of $100-200 \mathrm{~m} \mathrm{~s}^{-1}$ move across the footpoint of a dipole field (Fig. 15). Following the analytical treatment of Kan et al. (1983) the field-aligned current density can be expressed as

$$
j_{\|} \approx 4 \times 10^{-4}\left(\frac{\Sigma_{p}}{1 m h o}\right) \cdot\left(\frac{V_{n}}{100 \mathrm{~m} \mathrm{~s}^{-1}}\right) \cdot\left(\frac{B_{o}}{8 \mathrm{G}}\right) \cdot\left(\frac{200 \mathrm{~km}}{W}\right) A \mathrm{~m}^{-2}
$$

where $\Sigma_{p}$ is the height-integrated Pedersen conductivity, $B_{o}$ the surface magnetic field at the impact point and $\mathrm{W}$ is the typical width of the magnetic flux tube. Thus, the maximum value of the total parallel current would be as much as $1.6 \times 10^{10} \AA$ if the are length is $l \approx 2 \times 10^{7} \mathrm{~m}$.

In a dipole magnetic field geometry, the upward (from equator to pole) FAC $\left(j_{\|}\right)$is limited by the loss cone effect. That is, charged particles with pitch angles larger than the loss cone will be mirrored back to the equatorial region before reaching the dynamo 
or the load region near the planetary surface. To overcome such loss cone limitation, a voltage drop $(\Phi)$ along the magnetic field line must be developed to accelerate electrons so that their pitch angles could be reduced. From a consideration of the current flow of a thermal electron population with Maxwellian velocity distribution, a $j_{\|}-\Phi$ relation has been derived by Knight (1973) to be

$$
j_{\|}(o)=\left(\frac{B_{o}}{B_{m}}\right) \frac{e n_{e} V_{t h}}{\sqrt{2 \pi}}\left[1-\frac{\exp \left(-\delta \Phi / K T_{e}\right)}{1+\delta}\right]
$$

where $B_{m}$ is the magnetic field at the equator, $j_{\|}(0)$ is the parallel current density in the dynamo region, $n_{e}$ and $T_{e}$ are the number density and temperature of the ionospheric electrons in the magnetic flux tube, and the electron thermal velocity is $V_{t h}=\left(K T_{e} / m_{e}\right)^{\frac{1}{2}}$. Finally, $\delta=B_{m} /\left(B_{o}-B_{m}\right)$ is the numerical factor to account for the convergence of the magnetic field lines.

The combination of Eqs. (3.3) and (3.4) therefore provides a method to estimate the $\Phi$ value to be maintained by the impact-driven dynamo mechanism. As an example, for $B_{o}=8 G, B_{m}=0.5 G, n_{e}=10^{9} \mathrm{~cm}^{-3}, T_{e}=20 \mathrm{eV}$, we find that a voltage drop $\Phi \approx 70 \mathrm{~V}$ would be established if $j_{\|}^{(0)} \approx 0.4 \mathrm{~mA} / \mathrm{m}^{2}$. Since the energy dissipation rate due to particle acceleration is on the order of $P=j_{\|} \Phi A_{\|}$where $A_{\|}$is the effective area of the FAC we have $A_{\|} \approx 9 \times 10^{12} \mathrm{~m}^{2}$ from the HST observations and therefore $P \approx 3 \times 10^{12} \mathrm{~W}$ for the $\mathrm{K}$ impact (assuming an energy conversion factor of 10 ). As a result, our simplified consideration shows that the atmospheric dynamo effect is, in principle, a viable mechanism for the generation of the mid-altitude auroral emissions associated with the $\mathrm{K}$ impact.

To explain the morphology of the two bright UV arcs in the northern hemisphere and the two other arcs in the southern hemisphere after the K impact, Hill \& Dessler (1995) suggested that these are the result of the "snowplow" effect of the impact plumes as they re-entered atmosphere south of the impact site. Because the re-cntry speed was supersonic, a bow shock will form just ahead of the plume material which was separated from the ambient jovian atmosphere by a contact surface. In the reference frame of the impact plume, the jovian atmosphere will appear as a subsonic flow, which was diverted in the lateral direction after passing through the bow shock [Fig. 16(a)]. The shear velocity field in the lateral flow around the impact plume would then set up a pair of FAC of opposite directions [see Fig. 16(b)].

\subsection{Particle acceleration}

The ionospheric dynamo effect and the associated FAC systems are only one of the potential excitation mechanisms of the FUV mid-latitude auroral emissions and the impact-related infrared $\mathrm{H}_{3}^{+}$emissions. Other processes such as pitch angle scattering of the pre-existing charged particle population and/or in situ acceleration could also play a role. This is particularly true in the case of magnetospheric phenomena involving $\mathrm{MeV}$ electrons (i.e., the synchrotron radiations in decametric wavelengths) and the X-ray bursts associated with the $\mathrm{K}$ and $\mathrm{P} 2$ impacts.

The time scale for strong pitch angle scattering of energetic ions is given as (Gehrels and Stone 1983)

$$
\tau_{s}=\frac{\tau_{b} / 2}{\alpha_{o}^{2}} \approx 5 \mu^{-\frac{1}{2}} L^{\frac{11}{2}}[4-3 / L]^{\frac{1}{2}} \quad \mathrm{sec}
$$

where $\tau_{b}$ is the particle bounce period, $\alpha_{o}$ is the equatorial loss cone of the non-relativistic particles with magnetic moment $(\mu)$ in $\mathrm{MeV} / \mathrm{amu}$. If oxygen and sulfur ions with energies $>400 \mathrm{keV} / \mathrm{amu}$ were responsible for the $\mathrm{X}$-ray emissions of the $\mathrm{K}$ impact (Metzger 
(a)

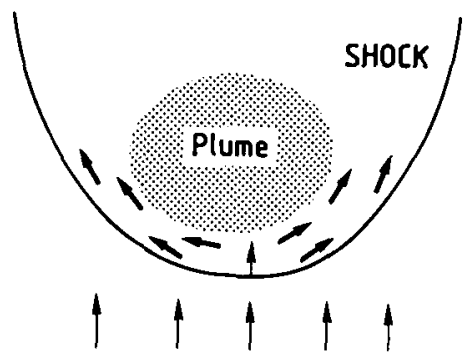

JOVIAN ATMOSPHERE (b)

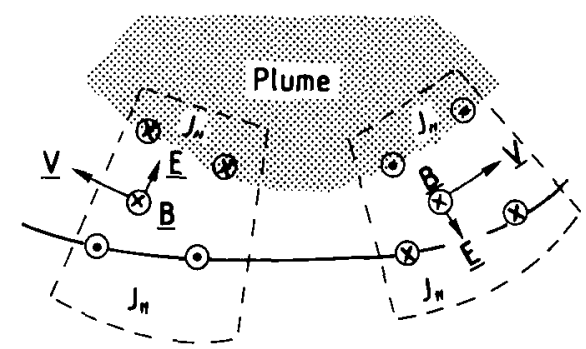

Figure 16. The Hill-Dessler model of the field-aligned current system driven by the atmospheric reentry of the " $K$ " impact plume (a) and the symmetric pair of dynamo regions (b). From Hill and Dessler (1995).

et al. 1983; Waite et al. 1994; 1995), the corresponding value of $T_{s}$ at $L \approx 6$ would be $\sim 28$ hours which is far too long in comparison with a time duration of just 30 seconds for the $\mathrm{K}$ event. Therefore, pitch angle scattering of energetic ions would not explain the X-ray outbursts even if the energy budget could be met by the trapped ion population. The pitch angle scattering time scale for $\mathrm{MeV}$ electrons is much shorter. But several major obstacles can be immediately identified. First, for electrons to supply the radiative power of $10^{10} \mathrm{~W}$ the corresponding mechanical power would have to be a factor of $10^{6}$ larger. This amounts to a total energy of $10^{26}$ ergs which is a significant fraction $(\sim 10 \%)$ of the total energy in the jovian magnetosphere according to the Dessler-Parker relation.

In their preliminary report, Waite et al. (1995) pointed out that the location of the $\mathrm{X}$-ray emission associated with the $\mathrm{K}$ impact was rather close to the Io flux tube which is known to be a source of decametric radio emissions (Goldstein and Goertz 1983) and infrared emission of the $\mathrm{H}_{3}^{+}$ions (Comerney et al. 1993). In addition, an intense flux of electrons with energies $\sim 0.5 \mathrm{MeV}$ was detected near the $\mathrm{L}$ shell of Io by the Trapped Radiation Detector on Pioneer 11 (Fillius 1976). However, it is not clear why the $\mathrm{K}$ impact at $\lambda_{I I I} \sim 270^{\circ}$ should have caused an energetic event at $\lambda_{I I I} \sim 180^{\circ}$, not to mention their separation in latitudinal angle.

As mentioned earlier, the X-ray emission presumably associated with the P2 impact provides yet another major puzzle. If this emission was situated in the polar cap region with magnetic field lines interconnected with the interplanetary magnetic field, it would be difficult to relate it to the P2 impact in the closed field region in the southern hemisphere. A careful magnetic field mapping might show that the X-ray hot spot could in fact be mapped back to a conjugate point in the southern hemisphere. The large angular separation between this X-ray hotspot and the conjugate footprint of the P2 or the Q1/Q2 fragment nevertheless remains problematic. In order to clarify this, it is essential to make detailed surveys of the jovian X-ray emissions using ROSAT and future spaceborne X-ray telescopes to document the spatial and temporal correlations with Io and 
the System Longitude $\lambda_{I I I}$. Probably after we have learned more about the statistical behaviour of the jovian X-ray aurora (do the emissions appear in localized regions and in bursts of short-duration?) we will be in a better position to pinpoint the origin of the impact-related events.

The interaction of the dust coma or the atmospheric impact of a comet fragment could cause impulsive disturbances to the magnetic flux tubes so that MHD waves could be excited to force random scattering of the charged particles. In this way, stochastic acceleration could be responsible for producing a population of energetic ions in the disturbed magnetic flux tubes. It is, therefore, possible that the UV emissions associated with the $\mathrm{K}$ impact could be related to this effect.

Brecht et al. (1995) have investigated electron acceleration by a collisionless shock wave generated by the comet impact in the jovian atmosphere. From the application of a theoretical model estimating the time required to accelerate electrons in the terrestrial inner Van Allen belt by the shock wave generated in the Starfish high altitude nuclear test, it was found that the mean time required to accelerate jovian magnetospheric electrons in the synchrotron radiation region from several keV to tens of $\mathrm{MeV}$ is $<1$ second. However, it is not certain whether the plasma environment surrounding the atmospheric fireball and subsequently the impact plume would permit the formation of the MHD shock as required in this model. Furthermore, de Pater et al. (1995) noted that there was no significant correlation between the longitudinal distribution of the synchrotron radiation enhancement and the impact sites.

\section{Future prospects}

Observations of the many facets of auroral and magnetospheric effects generated by the comet impacts have focused our attention on many hidden issues and provided us with a unique opportunity to examine the problems in a new light. For example, we have invoked radial diffusion, current systems, and stochastic acceleration to explain the excitation of anomalous UV and X-ray emissions observed. Many of these processes in the jovian magnetosphere are not well understood. The jovian system is known to be able to accelerate electrons and ions to high energies (MeV) very efficiently. But there is no definite idea of the basic mechanism(s). We know also very little about the electric current systems connecting the jovian ionosphere to the corotating magnetosphere on a global scale. As for radial diffusion, there exists no comprehensive theory describing the transport and energization of relativistic electrons in the inner magnetosphere which is characterized by strong longitudinal asymmetry. The important measurements of the impact-related microwave emissions described in Sec. 2.1 have demonstrated its need. We are, therefore, still at the very beginning of the understanding of the working of the jovian magnetosphere. The new measurements to be carried out by the Galileo mission in 1996 and 1997 will be most timely and useful in this respect.

At the same time, the continuing efforts in data analysis and interpretations of the observations of SL9-related effects will certainly bring more insights into the ionospheric and magnetospheric responses. Many interesting topics such as dust ablation in the highaltitude atmosphere (Moses 1992) and impact-induced aeronomical processes as discussed in Cravens (1994) will be investigated further. Detailed study will also be given to the excitation mechanisms of the mid-latitude UV aurora and the infrared $\mathrm{H}_{3}^{+}$emissions by theoretical modelling. As for the X-ray aurora, it is hoped that beyond the ROSAT era the XMM mission of ESA and other Earth-orbiting X-ray telescopes could be utilized to observe the corresponding spectral features and spatial and temporal variations. The 
ROSAT detection of the X-ray outbursts associated with the K and P2 impacts are most tantalizing.

Finally, the HST observations by Gerard et al. (1994a,b) and Clarke et al. (1995) have shown what a wealth of information is contained in the UV auroral emissions. Only by engaging in a systematic survey of the very dynamical behaviour of the jovian aurora, which is mapped to a wide range of plasma regions, will we be able to formulate a more complete picture of the jovian magnetosphere and hence its responses (i.e., the blinking aurora) to comet impacts or external disturbances of solar wind origin. Therefore, the saga of SL9 will continue.

It has been a great thrill to be able to participate in the magnetospheric study of the SL9 impact event at first hand. I must therefore thank Renée Prangé for drawing my attention, in the summer of 1993, to the pending comet collisions when I was still in Taiwan and for involving me in her HST observation project. I am also grateful to many colleagues who have provided me with useful information, stimulating discussions, and valuable advice in the preparation of this work. They are Nick Achilleos, Gilda Ballester, Scott Bolton, John Clarke, Imke de Pater, Alex Dessler, George Dulk, Sam Gulkis, Doyle Hall, Yolande Leblanc, Rita Schulz, Joachim Stüwe, Nick Thomas, and Hunter Waite. The assistance and efforts by Inge Gehne, Karin Peschke and Kim Schumann in the preparation of this manuscript are very much appreciated. I thank them all. The present work is supported in part by the Galileo Project of DARA. The quotation of John Minton is from the book "What Mad Pursuit" by Francis Crick.

\section{REFERENCES}

Acuna, M. H., Behannon, K. W. \& Connerney, J. E. P. 1983 Jupiter's magnetic field and magnetosphere. In Physics of the Jovian Magnetosphere, (ed. A. J. Dessler), pp. 1-50, Cambridge University Press.

Ballester, G. E., Harris, W. M., Gladstone, G. R. et al. 1995 Far-ultraviolet emissions from the impact sites of comet P/Shoemaker-Levy 9 with Jupiter. Geophys. Res. Lett. 22, 2425.

Belcher, J. W. 1983 The low-energy plasma in the Jovian magnetosphere, in Physics of the Jovian Magnetosphere, (ed. A. J. Dessler) pp. 68-105, Cambridge University Press.

Bolton, S. J. AND Thorne, R. M. 1995 Assessment of mechanisms for Jovian synchrotron variability associated with comet SL-9. Geophys. Res. Lett. 22, 1813.

Brecht, S. H., Pesses, M., Lyon, J. G., Gladd, N. T. and McDonald, S. W. 1995 An explanation of synchrotron radiation enhancement following the impact of Shoemaker-Levy 9 with Jupiter. Geophys. Res. Lett. 22, 1805.

Brice, N. AND IOANNidis, G. A. 1970 The magnetospheres of Jupiter and earth. Icarus 13, 173.

Brice, N. AND MCDonough, T. R. 1973 Jupiter's radiation belts. Icarus 18, 206.

Broadfoot, A. L., Belton, M. J. S., Takacs, P. Z., et al. 1979 Extreme ultarviolet observations from Voyager 1 encounter with Jupiter. Science 204, 979.

Brown, M. E., Moyer, E. J., Bouchez, A. H., and Spinrad, H. 1995 Comet ShoemakerLevy 9: No effect on the Io plasma torus, Geophys. Res. Lett. 22, 1833.

Brown, R. A., Pilcher, C. B. ANd STrobel, D. F. 1983 Spectrophotometric studies of the Io torus. In Physics of the Jovian Magnetosphere, (ed. A. J. Dessler, pp. 197-225. Cambridge University Press.

Clarke, J. T., Prangé, R., Ballester, G. E., et al. 1995 Hubble Space Telescope farultraviolet imaging of Jupiter during the impacts of comet Shoemaker-Levy 9. Science $\mathbf{2 6 7}, 1302$. 
Connerney, J. E. P., Baron, R., Satoh, T. And Owen, T. 1993 Images of excited $\mathrm{H}_{3}^{+}$at the foot of the Io flux tube in Jupiter's atmosphere. Science 262, 1035.

Cravens, T. E. 1994 Comet Shoemaker-Levy 9 impact with Jupiter; Aeronomical predictions. Geophys. Res. Lett. 21, 1075.

Crovisier, J., Biver, N., Bockelée-Morvan, D., Colom, P., Jorda, L. And Lellouch, E. 1995: Carbon monoxide outgassing from comet $\mathrm{P} /$ Schwassmann-Wachmann 1. Icarus 115, 213.

DE PATER, I. 1994 The effect of comet Shoemaker-Levy 9 on Jupiter synchrotron radiation. Geophys. Res. Lett. 21, 1071.

DE Pater, I. AND Goertz, C. K. 1990 Radial diffusion of energetic electrons and Jupiter's synchrotron radiation. I. Steady state solution. J. Geophys. Res. 95, 39.

DE PATER, I. AND Goertz, C. K. 1994 Radial diffusion models of energetic electrons and Jupiter's synchrotron radiation. II. Time variability. J. Geophys. Res. 99, 2271.

DE PATER, I. AND KLEIN, M. J. 1989 Time variability in Jupiter's synchrotron radiation. In Proc. Conference on Time Variable Phenomena in the Jovian System, pp. 139-150.

de PAter, I., Heiles, C., Wong, M., et al. 1995 Outburst of Jupiter's synchrotron radiation following the impact of comet P/Shoemaker-Levy 9. Science 268, 1879.

Dessler, A. J. 1980 Mass-injection rate from Io into the Io plasma torus. Icarus 44, 291.

Dessler, A. J. AND HiLl, T. W. 1994 Some interactions between dust from comet ShoemakerLevy 9 and Jupiter. Geophys. Res. Lett. 21, 1043.

Dougherty, M. K., Southwood, D. J., BALOgh, A. AND Smith, E. J. 1993 Field-aligned currents in the jovian magnetosphere during the Ulysses flyby. Planet. Space Sci. 41, 291.

DulK, G. A. AND LEBLANC, Y. 1994 Changes in jupiter's synchrotron radiation belts during and after SL-9 impacts. In Proc. European SL-9/Jupiter Workshop (eds. R. West and H. Böhnhardt), p. 381. ESO.

Dulk, G. A., Leblanc, Y., AND Hunstead, R. W. 1995 Flux and images of Jupiter at 13, 22 and $36 \mathrm{~cm}$ before, during and after SL-9 impacts. Geophys. Res. Lett. 22, 1789.

Farrell, W. M., Kaiser, M. L., Desch, M. D. AND MacDowall, R. J. 1994 Possible radio wave precursors associated with the comet Shoemaker-Levy 9/Jupiter impacts. Geophys. Res. Lett. 21, 1067.

Fillius, R. 1976 The trapped radiation belts of Jupiter. In Jupiter (ed. T. Gehrels), pp. 896-927. University of Arizona Press.

Gehrels, N. AND STONE, E C. 1983 Energetic oxygen and sulfur ions in the Jovian magnetosphere and their contribution to the auroral excitation. J. Geophys. Res. 88, 5537.

Gerard, J. C., Dols, V., Prangé, R., And Paresce, F. 1994a Morphology and time variation of the Jovian far uv. Planet. Space Sci. 42, 905.

Gerard, J. C., Grodent, D., Prangé, R., et al. 1994b A remarkable auroral event on Jupiter observed in the ultraviolet with the Hubble Space Telescope aurora: Hubble Space Telescope observations. Science 266, 1675.

Goldstein, M. L. AND GoERTz, C. K. 1983 Theories of radio emissions and plasma waves. In Physics of the Jovian Magnetosphere pp. 317-352. Cambridge University Press.

Hall, D. T., Gladstone, G. R., Herbert, F., Lieu, R., and Thomas, N. 1995 Io torus EUV emissions during the comet Shoemaker-Levy 9 impacts. Geophys. Res. Lett. 22, 3441.

Herbert, F. 1994 The impact of comet Shoemaker-Lev 9 on the Jovian magnetosphere. Geophys. Res. Lett. 21, 1047.

Herbert, F., SANDEL, B. R. AND Broadfoot, A. L. 1987 Observations of the jovian UV aurora by Voyager. J. Geophys. Res. $92,3141$.

HiLl, J. R. AND MENDIS, D. A. 1980 On the origin of striae in cometary dust tails. Astrophys. $J$. 242, 395.

Hill, T. W. AND Dessler, A. J. 1995 Midlatitude Jovian aurora produced by the impact of comet Shoemaker-Levy 9. Geophys. Res. Lett. 22, 1817. 
HoOD, L. L. 1993 Long-term changes in jovian synchrotron radio emission: intrinsic variations or effects of viewing geometry. J. Geophys. Res. 98, 5769.

Horanyi, M. H. 1994 New Jovian rings. Geophys. Res. Lett. 21, 1039.

IP, W.-H. 1994 Time variations of the Jovian synchrotron radiation following the collisional impacts of comet Shoemaker-Levy 9. Planet. Space Sci. 42, 527.

IP, W.-H. 1995a Time variations of the Jovian synchrotron radiation following the collisional impacts of comet Shoemaker-Levy 9: 2 . flux enhancement induced by neutral atmospheric turbulence. Planet. Space Sci. 43, 221.

IP, W.-H. 1995b On particle acceleration by the impact-driven field-aligned current systems. Icarus, submitted.

IP, W.-H. AND Prangé, R. 1994 On possible magnetospheric dust interactions of comet Shoemaker-Levy 9 at Jupiter. Geophys. Res. Lett. 21, 1051.

Kan, J. R., Akasofu, S.-I. ANd LeE, L. C. 1983 A dynamo theory of solar flares. Solar Phys. 84, 153.

KellogG, P. J. 1994 Plasma effects on the interaciton of a comet with Jupiter. Geophys. Res. Lett. 21, 1055.

KLEIN, M. J., GulkIS, S., Bolton, S. J. 1995 Changes in Jupiter's 13-cm synchrotron radio emission following the impact of comet Shoemaker-Levy 9. Geophys. Res. Lett. 22, 1797.

KNIGHT, S. 1973 Parallel electric field. Planet. Space Sci. 21, 741.

Lanzerotti, L. J., Armstrong, T. P., Maclennan, C. G., et al. 1993 Measurements of hot plasmas in the magnetosphere of Jupiter. Planet. Space Sci. 41, 893.

Leblanc, Y. AND DulK, G. A. 1995 Changes in brightness of Jupiter's radiation belt at 13 and $22 \mathrm{~cm}$ during and after impacts of comet SL-9. Geophys. Res. Lett. 22, 1793.

Livengood, T. A., Strobel, D. F. AND Moos, H. W. 1990 Jupiter's north and south polar aurorae with IUE data. J. Geophys. Res. 95, 10375.

McGrath, M. A., Hall, D. T., Matheson, P. L., et al. 1995. Response of the Io plasma torus to comet Shoemaker-Levy 9. Science 267, 1313.

MCGregor, P. J., Nicholson, P. D. AND Allen, M. G. 1995 CASPIR observations of the collision of comet Shoemaker-Levy 9. Icarus submitted.

Mendis, D. A., Hill, J. R., Houpis, H. F., AND WhipPle, E. C. 1981 On the electrostatic charging of the cometary nucleus. Astrophys. J. 249, 787.

Metzger, A. E., Gilman, D. A., Luthey, J. L. et al. 1983 The detection of x-rays from Jupiter. J. Geophys. Res. 88, 7731.

Miller, S., Achilleos, N., Dinelli, B. M., et al. 1995 The effect of the impact of comet Shoemaker-Levy 9 on Jupiter's aurora. Geophys. Res. Lett. 22, 1629.

Moses, J. 1992 Meteoroid ablation in Neptune's atmosphere. Icarus 99, 368.

Prangé, R., Rego, D. And Gerard, J. C. 1995a Auroral Lyman alpha and H2 bands from the giant planets 2. Effect of the anisotropy of the precipitating particles on the interpretation of the "color ratio." J. Geophys. Res. 100, E4, 7513.

Prangé, R., Engle, I., Clarke, J. T., et al. 1995b Auroral signature of comet SL9 in the Jovian magnetosphere. Science 267, 1317.

Prangé, R., Engle, I., Dunlop, M., Maurice, S., And Rego, D. 1995c Magnetic mapping of auroral signatures of comet SL9 in the Jovian magnetosphere. Geophys. Res. Lett., submitted.

SENAY, M. AND JEWITT, D. 1994 Coma formation driven by carbon monoxide release from Comet Schwassmann-Wachmann, I., Nature 371, 229.

ShemANSKY, D. E. 1988 Energy branching in the Io plasma torus: The failure of neutral cloud theory. J. Geophys. Res. 93, 1773.

Schulz, M. And Lanzerotti, L. J. 1974 Particle Diffusion in the Radiation Belt. SpringerVerlag. 
Schulz, R., Encrenaz, Th., Stüwe, J. A., and Wiedemann, G. 1995 Near-IR emissions in the upper Jovian atmosphere after SL9 impact: Indications of possible northern counterpart. Geophys. Res. Lett., 22, 2421.

ThOMSEN, M. F. 1979 Jovian magnetosphere-satellite interactions: aspects of energetic charged particle loss. Rev. Geophys. Space Phys. 17, 369.

VAsyliunas, V. M. AND Dessler, A. J. 1981 The magnetic anomaly model of the Jovian magnetosphere: A post-Voyager assessment. J. Geophys. Res. 86, 8435.

Waite, J. H. Jr., Bagenal, F., Seward, C. et al. 1994 ROSAT observations of the Jupiter aurora. J. Geophys. Res. 99, 14799.

Waite, J. H. JR., Gladstone, G. R., Franke, K., et al. 1995 ROSAT observations of X-ray emissions from Jupiter during the impact of comet Shoemaker-Levy 9. Science, 268, 1598.

Weaver, H. A., A'Hearn, M. F., Arpigny, C. et al. 1995 The Hubble Space Telescope oberving campaign on comet P/Shoemaker-Levy 9. Science 267, 1282. 